\title{
Descending Modulation of Laryngeal Vagal Sensory Processing in the Brainstem Orchestrated by the Submedius Thalamic Nucleus
}

\author{
${ }^{D}$ Stuart B. Mazzone, ${ }^{1}$ Tara G. Bautista, ${ }^{1}$ Anthony J. M. Verberne, ${ }^{2}$ Matthew W. Trewella, ${ }^{1}{ }^{\circledR}$ Michael J. Farrell, ${ }^{3}$ \\ and ${ }^{\circledR}$ Alice E. McGovern ${ }^{1}$ \\ ${ }^{1}$ Department of Anatomy and Neuroscience, The University of Melbourne, Parkville 3010, Victoria, Australia, ${ }^{2}$ Clinical Pharmacology and \\ Therapeutics Unit, Department of Medicine, Austin Health, The University of Melbourne, Heidelberg 3084, Victoria, Australia, and ${ }^{3}$ Department of \\ Medical Imaging and Radiation Sciences and Monash Biomedical Imaging, Monash University, Clayton 3800, Victoria, Australia
}

The nodose and jugular vagal ganglia supply sensory innervation to the airways and lungs. Jugular vagal airway sensory neurons wire into a brainstem circuit with ascending projections into the submedius thalamic nucleus (SubM) and ventrolateral orbital cortex (VLO), regions known to regulate the endogenous analgesia system. Here we investigate whether the SubMVLO circuit exerts descending regulation over airway vagal reflexes in male and female rats using a range of neuroanatomical tracing, reflex physiology, and chemogenetic techniques. Anterograde and retrograde neuroanatomical tracing confirmed the connectivity of the SubM and VLO. Laryngeal stimulation in anesthetized rats reduced respiration, a reflex that was potently inhibited by activation of SubM. Conversely, inhibition of SubM potentiated laryngeal reflex responses, while prior lesions of VLO abolished the effects of SubM stimulation. In conscious rats, selective chemogenetic activation of SubM neurons specifically projecting to VLO significantly inhibited respiratory responses evoked by inhalation of the nociceptor stimulant capsaicin. Jugular vagal inputs to SubM via the medullary paratrigeminal nucleus were confirmed using anterograde transsynaptic conditional herpes viral tracing. Respiratory responses evoked by microinjections of capsaicin into the paratrigeminal nucleus were significantly attenuated by SubM stimulation, whereas those evoked via the nucleus of the solitary tract were unaltered. These data suggest that jugular vagal sensory pathways input to a nociceptive thalamocortical circuit capable of regulating jugular sensory processing in the medulla. This circuit organization suggests an intersection between vagal sensory pathways and the endogenous analgesia system, potentially important for understanding vagal sensory processing in health and mechanisms of hypersensitivity in disease.

Key words: airway innervation; jugular vagal ganglia; respiratory; submedius thalamic nucleus; vagus nerve; ventrolateral orbital cortex

\section{Significance Statement}

Jugular vagal sensory pathways are increasingly recognized for their important role in defensive respiratory responses evoked from the airways. Jugular ganglia neurons wire into a central circuit that is notable for overlapping with somatosensory processing networks in the brain rather than the viscerosensory circuits in receipt of inputs from the nodose vagal ganglia. Here we demonstrate a novel and functionally relevant example of intersection between vagal and somatosensory processing in the brain. The findings of the study offer new insights into interactions between vagal and spinal sensory processing, including the medullary targets of the endogenous analgesia system, and offer new insights into the central processes involved in airway defense in health and disease.

Received Sep. 14, 2020; revised 0ct. 20, 2020; accepted 0ct. 21, 2020.

Author contributions: S.B.M. and A.E.M. designed research; T.G.B., A.J.M.V., M.W.T., M.J.F., and A.E.M. performed research; T.G.B., A.J.M.V., M.J.F., and A.E.M. analyzed data; S.B.M. and A.E.M. wrote the paper.

A.E.M. is currently funded by National Health and Medical Research Council (NHMRC) of Australia

Fellowship APP1121376. This research was supported by NHMRC Grant 1078943 to S.B.M.

The authors declare no competing financial interests.

Correspondence should be addressed to Alice E. McGovern at alice.mcgovern@unimelb.edu.au..

https://doi.org/10.1523/JNEUROSCI.2430-20.2020

Copyright $\odot 2020$ the authors

\section{Introduction}

The mammalian airways and lungs are innervated by populations of sensory nerve fibers that are mostly vagal in origin. Two different vagal ganglia, named the nodose and jugular ganglia, supply these sensory fibers and when activated by airway stimuli they initiate a range of sensations and motor responses that regulate breathing, airway clearance, and airway patency. Importantly, nodose and jugular vagal ganglia neurons are embryologically 
distinct, with the former representing the quintessential vagal visceral sensors and the latter resembling more the somatosensory neurons of the dorsal root ganglia (Nassenstein et al., 2010; McGovern et al., 2015a; Kupari et al., 2019; Mazzone et al., 2020). Within the airway tree, a transition exists from a substantial jugular innervation present in the larynx and proximal airways to the predominantly nodose innervation present in the intrapulmonary airways and lung parenchyma. This transition of vagal sensory innervation is suggestive of a shift from somatosensory processes contributing to laryngeal control to more traditional viscerosensory regulation of the lower airways and lungs.

Responses evoked through vagal sensory innervation of the airways are regulated via reasonably well defined reflex circuits in the brainstem and less well defined networks in the higher brain. Importantly, we have previously shown that the brainstem pathways contributing to nodose and jugular vagal sensory-evoked responses are not the same. Nodose sensory neurons innervating the airways, like most other vagal visceral afferents, terminate centrally in the nucleus of the solitary tract (NTS), whereas neurons of the jugular vagal ganglia terminate within the trigeminal nuclei, notably in and around the paratrigeminal nucleus $(\mathrm{Pa} 5)$ in the caudal medulla (Driessen et al., 2015; McGovern et al., 2015b; Bautista et al., 2019; Farrell et al., 2020; Kim et al., 2020). These termination sites, and the resultant networks in receipt of outputs from NTS and Pa5 neurons, provide further evidence of the dual viscerosensory and somatosensory controls at play in the airways and lungs.

The behavioral responses that accompany airway stimulation involve network activity in subcortical and cortical brain regions that indirectly receive airway vagal sensory inputs. Our human brain imaging studies demonstrate multiple networks for discriminative and affective processing of airway sensory inputs, but additionally have identified higher brain processes capable of modulating airway vagal sensory processing, including modulation through placebo conditioning and heterotopic painful stimuli (conditioned pain modulation; Mazzone et al., 2009; Farrell et al., 2012; Leech et al., 2012, 2013; Abubakar et al., 2020). Similar inhibitory controls have been described modulating noxious spinal somatosensory processing leading to the perception of pain. Indeed, a well described endogenous neural system, referred to as the "endogenous analgesia system" or the "descending antinociceptive/analgesic pathway," can modulate sensory inputs to second-order neurons at the level of the spinal dorsal horn. The network organization of this system is relatively well described, and the higher-order controls include multiple subcortical and prefrontal pathways that ultimately regulate descending inputs to the spinal dorsal horn to tune spinal sensory processing (Basbaum and Fields, 1984; Dubner and Ren, 2013). One pathway regulating the descending analgesia system involves the activation of the submedius thalamic nucleus (SubM) and its connectivity to the ventrolateral orbital cortex (VLO; Coffield et al., 1992; Yoshida et al., 1992; Zhang et al., 1998, 1999; Tang et al., 2009; Kuramoto et al., 2017). Notably, we have previously shown that airway-specific vagal sensory inputs to the brainstem reach the SubM via connectivity specifically within in the medullary Pa5 (and not the NTS), suggestive that jugular vagal pathways may also intersect with the descending analgesia system (McGovern et al., 2015b). Given these combined observations, we set out in the present study to investigate whether the SubM is capable of orchestrating regulatory controls over laryngeal vagal sensory processing in the brainstem.

\section{Materials and Methods}

\section{Animals}

Experiments were approved by an accredited institutional Animal Ethics Committee and were conducted on adult Sprague Dawley rats of either sex (weight range, 300-500 g; $N=109$ ). Animals were housed on a $12 \mathrm{~h}$ light/dark cycle and given ad libitum access to food and water. No statistical differences were observed between the measured responses of male and female rats in initial experiments, and, in turn, the data were pooled for all subsequent statistical comparisons.

\section{Physiologic studies in anesthetized rats}

Rats were anesthetized with urethane (1.5 g/kg, i.p.), the level of which was determined to be adequate by assessing the palpebral and limb withdrawal reflexes. Animals were placed supine on a thermostatically controlled heating pad set at $36.5^{\circ} \mathrm{C}$, and body temperature was maintained via feedback from a rectal probe. A midline incision was made along the ventral surface of the neck to expose the larynx, trachea and underlying nerves and blood vessels. The left carotid artery was cannulated using polyethylene tubing (internal diameter, $0.5 \mathrm{~mm}$; outer diameter, $0.9 \mathrm{~mm}$ ) attached to a pressure transducer filled with heparinized saline $(50 \mathrm{U} / \mathrm{ml}$; Sigma-Aldrich) to measure arterial blood pressure (ABP). The distal extrathoracic trachea was cannulated and connected via a side port to a pressure transducer to measure the tracheal pressure and changes associated with spontaneous respiration. Output from pressure transducers was digitized and recorded (LabScribe, iWorx). A midline incision was made through the larynx, and a platinum bipolar stimulating electrode was placed on the mucosal surface (Fig. 1A). After a 20 min stabilization period, electrical stimulation (S48 square pulse stimulator, Grass Instruments) of the larynx was performed to activate the peripheral terminals of vagal afferents innervating this site (Driessen et al., 2015, 2020). A voltage response curve was initially conducted by delivering increasing voltages $(0.5-5 \mathrm{~V} ; 0.5 \mathrm{~V}$ increments at 2 min intervals) to the larynx, at a constant maximum stimulating frequency $(32 \mathrm{~Hz})$, pulse duration $(1 \mathrm{~ms})$, and train duration $(10 \mathrm{~s})$. In each preparation, the optimum voltage to elicit complete apneic responses, mimicking the laryngeal chemoreflex evoked by aspiration or chemical activation of vagal laryngeal afferents (Xia et al., 2008), was used to conduct a frequency response analysis by sequentially increasing the stimulation frequency $(1-32 \mathrm{~Hz})$. Across all experiments, the average voltage used was $1.85 \pm 0.12 \mathrm{~V}$ (range, $1-3 \mathrm{~V}$ ), which is expected to activate all subtypes of sensory terminals innervating the larynx (McAllen et al., 2018). The specificity of vagal sensory pathways mediating respiratory effects was confirmed after unilateral and bilateral vagotomy. Following this initial baseline electrical stimulation of the larynx, rats were placed onto a stereotaxic frame (model 940, David Kopf Instruments), the skull exposed, and burr holes drilled at the corresponding stereotaxic coordinates for bilateral microinjections into the SubM [anteroposterior (AP), -2.75 $\mathrm{mm}$; mediolateral (ML), $\pm 2.00 \mathrm{~mm}\left(12^{\circ}\right.$ angle); dorsoventral (DV), $-7.10 \mathrm{~mm}$, relative to bregma] of vehicle (0.9\% saline; $250 \mathrm{nl}), 5-\mathrm{HT}$ ( $5 \mu \mathrm{g}$ of 5-HT-HCl, $150 \mathrm{nl}$; Sigma-Aldrich) to stimulate analgesic promoting SubM neurons via $5-\mathrm{HT}_{1 \mathrm{~A}}$ and $5-\mathrm{HT}_{2}$ receptors, or the generic neuronal inhibitor muscimol (10 ng, $150 \mathrm{nl}$; Sigma-Aldrich) to inhibit SubM-mediated analgesic neurons (Xiao et al., 1999, 2005a,b; Jia et al., 2004). Microinjections of pharmacological agents were performed using glass-pulled micropipettes (tip diameter, $\sim 20 \mu \mathrm{m}$ ) connected to a microprocessor-controlled picopump (model PV820, World Precision Instruments). Solutions were combined with red fluorescent beads (Lumofluor) to allow easy visualization of injection sites. Injections outside of the SubM target site, into adjacent nuclei, served as control experiments to ensure that evoked responses were mediated by the SubM. In some experiments, an electrolytic lesion of the ventrolateral orbital cortex $(\mathrm{AP},+3.50 \mathrm{~mm}$; $\mathrm{ML}, \pm 2.50 \mathrm{~mm}$; DV, $-5.10 \mathrm{~mm}$ ) was performed using an LMD Lesion Making Device (15 s, $1 \mathrm{~mA}$, DC constant current; Ugo Basile), with sham lesions involving the insertion of the electrode but without delivering any current. After microinjections, rats were immediately returned to a supine position and the frequency response to electrical stimulation of the larynx reassessed in a paired experimental design. In all instances, 


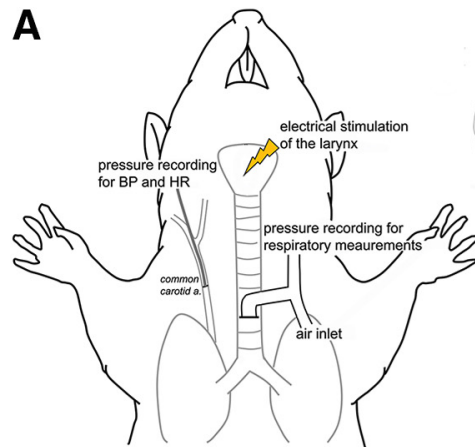

D

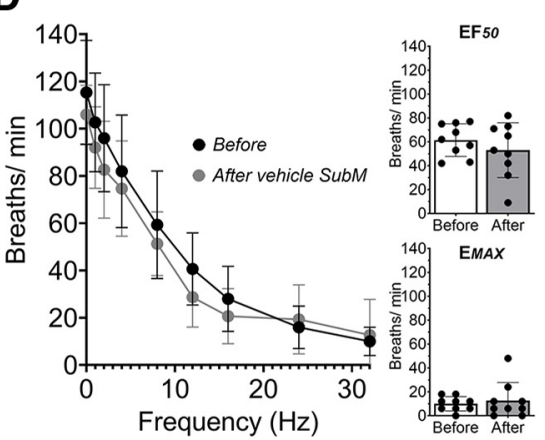

G

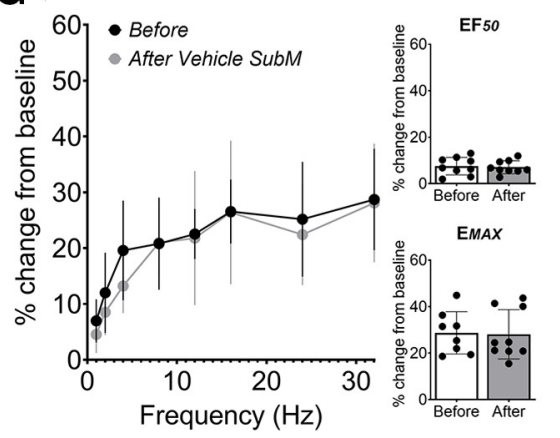

B
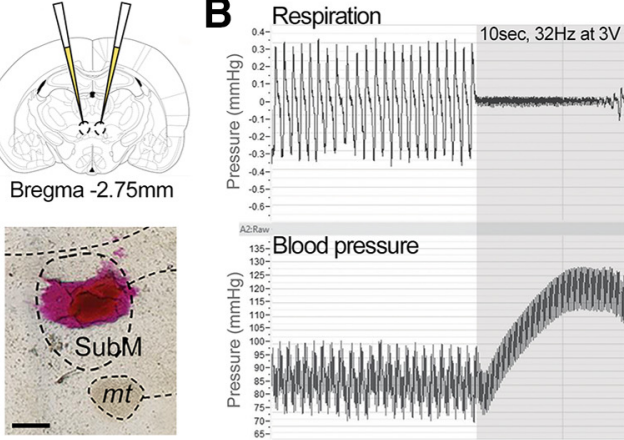

E
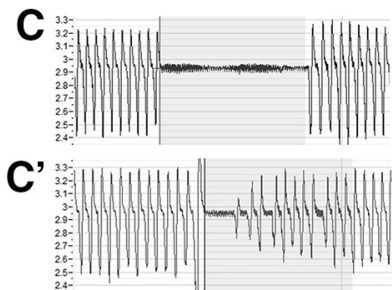

C"

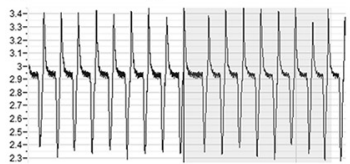

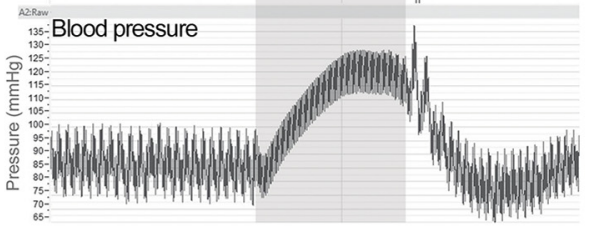

F
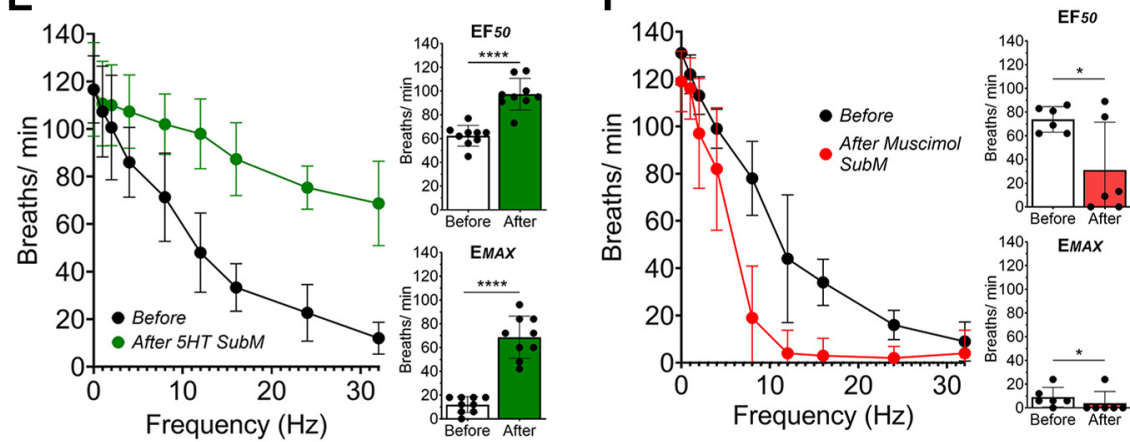

H

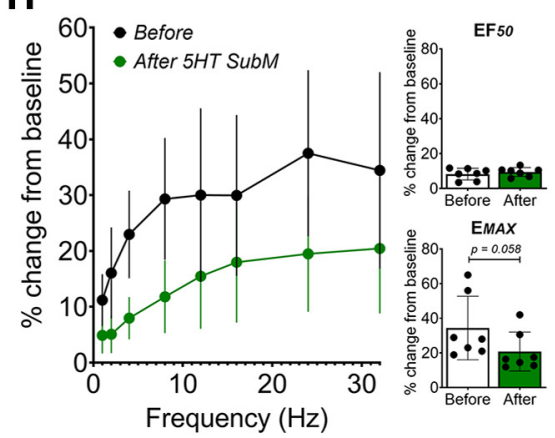

I

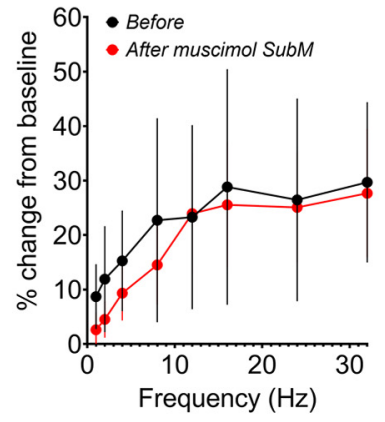

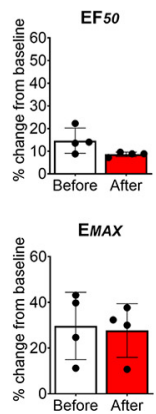

Figure 1. Pharmacological modulation of the submedius thalamic nucleus regulates laryngeal-evoked respiratory reflexes. $A$, Anesthetized respiratory physiology setup and example of a SubM microinjection site. $\boldsymbol{B}$, Electrical stimulation of the larynx results in a frequency-dependent slowing of respiration accompanied by hypertension. $\mathbf{C}-\boldsymbol{C}^{\prime \prime}$, This respiratory effect $(\boldsymbol{C})$ is partially reduced by unilateral vagotomy $\left(\boldsymbol{C}^{\prime}\right)$ and completely abolished following bilateral vagotomy $\left(\boldsymbol{C}^{\prime \prime}\right)$. $\mathbf{D}-\boldsymbol{F}$, Stimulus frequency-dependent responses are assessed before (Before) bilateral microinjection of pharmacological agents into the SubM and repeated afterward (After). $\boldsymbol{D}$, Stimulation-evoked changes in respiratory rate before and after microinjection of vehicle ( $N=9$ ) into the SubM. $\boldsymbol{E}$, Stimulation-evoked changes in respiratory rate before and after microinjection of 5-HT $(N=9)$ into the SubM. $\boldsymbol{F}$, Stimulation-evoked changes in respiratory rate before and after microinjection of muscimol $(N=6)$ into the SubM. $\mathbf{G}-\mathbf{I}$, Blood pressure stimulus-response curves before and after microinjection of vehicle $(N=9 ; \boldsymbol{G})$, microinjection of 5-HT $(N=9 ; \boldsymbol{H})$, and microinjection of muscimol $(N=6)$ into the SubM $(\boldsymbol{I})$. *p $<0.05$, ****p $<0.0001$, two-tailed paired $t$ test $(\boldsymbol{F}-\boldsymbol{I})$. Scale bar, $\boldsymbol{A}, 300 \mu \mathrm{m}$. $m t$, Mamillary tract. Gray box $(\boldsymbol{B}, \boldsymbol{C})$ denotes a $10 \mathrm{~s}$ period of maximum electrical stimulus intensity.

the second series of laryngeal stimuli was completed within a period of $30 \mathrm{~min}$ from the first microinjection.

In some experiments, bilateral microinjections of the nociceptor stimulant capsaicin were performed into the medullary NTS and Pa5, instead of electrical stimulations of the larynx, to activate the central terminals of vagal afferent neurons projecting to these discrete sites (McGovern et al., 2012, 2015a,b; Driessen et al., 2015; Mazzone and Undem, 2016; Kim et al., 2020). In these experiments, rats were set up to record respiration and blood pressure parameters, following which they were placed onto a stereotaxic frame and either $5-\mathrm{HT}$ or vehicle was bilaterally microinjected into the SubM (as above). To access the medulla, the head was angled at $45^{\circ}$ and a midline incision was made through the skin of the animal, the posterior neck muscles, and the dura mater between the occipital bone and $\mathrm{C} 1$ vertebra. Using obex as a reference point ( $\sim 0.5 \mathrm{~mm}$ rostral to the calamus scriptorius), microinjections of capsaicin $(0.5 \mu \mathrm{M}, 150 \mathrm{nl}$; Sigma-Aldrich) were made into either the caudal NTS ( $0 \mathrm{~mm}$ level to obex, $0.3 \mathrm{~mm}$ lateral to the midline, and 0.5 $\mathrm{mm}$ below the medullary surface) or Pa5 (0.5 $\mathrm{mm}$ rostral to obex, 2-3 $\mathrm{mm}$ lateral to the midline, and $0.5 \mathrm{~mm}$ below the medullary surface).
Respiration and blood pressure parameters were monitored for $30 \mathrm{~min}$ after capsaicin microinjection.

Neuronal tracer and viral vector injections

Rats were anesthetized with isoflurane (4\% induction and $2 \%$ maintenance in oxygen) via a nose cone and placed onto a stereotaxic frame as described above. The adequacy of anesthesia and body temperature was monitored as described above. Microinjections were made using the same stereotaxic coordinates described above. SubM-VLO connectivity was assessed by retrograde tracing from the VLO using green fluorescent retrobeads $(N=4 ; 250 \mathrm{nl}$; Lumofluor) or anterograde tracing from the SubM using biotin dextran amine (BDA; $10 \% \mathrm{w} / \mathrm{v}, 150 \mathrm{nl}$; $N=4$; SigmaAldrich). For conscious behavioral experiments, we opted to target specifically the VLO-projecting SubM neurons by bilaterally microinjecting adeno-associated virus (AAV) retrograde-pkg-Cre $(250 \mathrm{nl}$; catalog \#24593, Addgene) into the VLO and either AAV8-hSyn-DiO-hM3D (Gq)-mCherry ( $N=10 ; 150 \mathrm{nl}$; catalog \#44361, Addgene) or control vector AAV8-hSyn-DiO-mCherry ( $N=9 ; 150 \mathrm{nl}$; catalog \#50459, Addgene) bilaterally into the SubM. For anterograde transsynaptic neuronal 
tracing, $15 \mu \mathrm{l}$ of the conditional HSV1-H129 $9_{\text {floxed }}\left(1 \times 10^{9} \mathrm{pfu} / \mathrm{ml}\right)$ was injected into the airway lumen of rats $(N=6)$ that had previously received bilateral microinjections of the AAV2 pkg-Cre $\left(250 \mathrm{nl} ; 1 \times 10^{11}\right.$ viral genomes $/ \mathrm{ml}$ ) into the $\mathrm{Pa} 5$ (see above for coordinates; see Fig. $4 E$ ). Following the completion of microinjections, sterile bone wax was applied to cover the holes in the top of the skull, incisions were sutured, and rats received subcutaneous injections of meloxicam $(1 \mathrm{mg} / \mathrm{kg})$ and 1 $\mathrm{ml}$ of $0.9 \%$ sterile saline. Rats were allowed to recover for $7 \mathrm{~d}$ for conventional tracing studies, 5-6d for transsynaptic viral tracing studies (McGovern et al., 2015a,b), and $28 \mathrm{~d}$ for conscious respiratory behavioral studies.

\section{Conscious respiratory studies}

Conscious unrestrained rats were exposed to an aerosolized respiratory irritant in a four-site Buxco whole-body plethysmography system. The experimental design consisted of a $29 \mathrm{~d}$ protocol whereby rats were microinjected with either the $\mathrm{hM} 3 \mathrm{D}(\mathrm{Gq})$ or control vector (see above; day 1 ), were allowed to recover for $23 \mathrm{~d}$, then acclimatized to the plethysmography chambers $(1 \mathrm{~h} / \mathrm{d}$ ) without aerosolized challenge (days 23,25 , and 27). On challenge day (day 29), all rats were administered the hM3D (Gq) ligand clozapine $\mathrm{N}$-oxide (CNO; $3 \mathrm{mg} / \mathrm{kg}$, i.p.), following which they were placed in the chambers for $30 \mathrm{~min}$ before receiving an initial aerosolization of saline (vehicle) followed by increasing challenge doses of capsaicin $(0.3,3,30,100$, and $300 \mu \mathrm{M})$. Challenge substances were nebulized (Buxco Control-10 Aerosol Delivery Unit 10 LPM, Aerogen nebulizer unit AG-AL1000 with filter cap of $3.1 \mu \mathrm{m}$ particle size) as $1 \mathrm{ml}$ solutions over $5 \mathrm{~min}$, at an air flow velocity of $5 \mathrm{~L} / \mathrm{min}$, and at $60 \%$ nebulizer duty cycle. Challenge substances were nebulized for $5 \mathrm{~min}$, followed by a $5 \mathrm{~min}$ postchallenge response period. A further $5 \mathrm{~min}$ was allowed between each challenge dose to allow respiratory parameters and behaviors to return to baseline. Rats were left in the chambers for $30 \mathrm{~min}$ after the completion of the capsaicin challenges to allow for adequate time for induction of c-Fos post CNO. Two additional control groups of rats, no surgery $+\mathrm{CNO}(N=6)$ and no surgery and no $\mathrm{CNO}(N=6)$, underwent the same respiratory challenge protocol.

\section{Tissue harvest and histologic analyses}

Following completion of conscious, physiological, or tracing studies, rats were overdosed with sodium pentobarbital $(100 \mathrm{mg} / \mathrm{kg}$, i.p.) and transcardially perfused with $200 \mathrm{ml}$ of $0.1 \mathrm{M} \mathrm{PBS}$, pH 7.4, and $200 \mathrm{ml}$ of $4 \%$ paraformaldehyde (PFA) in $0.1 \mathrm{M}$ PBS. Brains were dissected and postfixed overnight in $4 \%$ PFA before being cryoprotected in $25 \%$ sucrose at $4^{\circ} \mathrm{C}$. Tissues were frozen in OCT (optimal cutting temperature) embedding compound, and $50 \mu \mathrm{m}$ cryostat cut sections encompassing the areas of interest were collected serially into $0.1 \mathrm{M}$ PBS. Sections from the physiological studies were mounted directly onto gelatin-coated slides and coverslipped to assess SubM injection sites and VLO lesions. All fluorescent immunohistochemistry was performed at room temperature. Brain sections were rinsed then blocked in $10 \%$ donkey serum in $0.1 \mathrm{M}$ PBS for 1 $\mathrm{h}$ before incubation for $48 \mathrm{~h}$ in the primary antibody of interest: rabbit anti-calbindin D-28k (1:1000; catalog \#CB-38a, Swant); mouse anti-NeuN (1:1000; catalog \#MAB377, Merck Millipore); rabbit anti-c-Fos (1:2000; catalog \#26209, Immunostar). All antibodies were diluted in antibody diluent ( $2 \%$ donkey serum and $0.3 \%$ Triton X-100 in $0.1 \mathrm{~m}$ PBS). After the required incubation, sections were washed three times with $0.1 \mathrm{M}$ PBS for $20 \mathrm{~min}$ each then incubated for $1 \mathrm{~h}$ in appropriate fluorescently conjugated secondary antibodies (1:500; Thermo Fisher Scientific). BDA traced sections were incubated in streptavidin-Alexa Fluor 594 conjugate (1:500; catalog \#S32356, Thermo Fisher Scientific) for $1 \mathrm{~h}$. Immunostained sections were kept in order, mounted onto gelatin-coated slides, and coverslipped with an antifade mounting media (Fluoroshield, catalog \#F6182, Sigma-Aldrich). All tissues were visualized under the appropriate filter cube using a Leica DM6B LED Microscope, and images were captured using a Leica DFC7000T camera. Representative photomicrographs were assembled in Adobe Photoshop CS6.

\section{Experimental design and statistical analyses}

All statistical comparisons were made in Prism software (version 7.03; GraphPad Software). For Student's $t$ test and ANOVA all $T$ and $p$, and $F$ and $p$ values for the interaction effects and individual treatment effects, respectively, are reported either in the text or in corresponding figure legends. Degrees of freedom for each $F$ value are designated by subscripts as per convention. Data are reported as the mean $\pm \mathrm{SD}$, unless stated otherwise; and differences were considered significant at $p<0.05$.

Anesthetized physiological respiratory studies. Respiratory rate, blood pressure, and heart rate were calculated from the raw chart recordings over a $10 \mathrm{~s}$ period, 2 min before any stimulation (baseline) and then again during each laryngeal stimulation (evoked response), and multiplied by 6 to determine the equivalent per minute values, and respiration was plotted as the mean \pm SD for all groups. For capsaicin medullary microinjection experiments, respiratory rate, blood pressure, and heart rate were collected from the raw chart recordings at $60 \mathrm{~s}$ intervals over a period of $10 \mathrm{~min}$ after the first microinjection of capsaicin. Baseline measurements were determined as the $60 \mathrm{~s}$ period before capsaicin microinjection with data presented as the percentage change relative to baseline and plotted as the mean $\pm \mathrm{SD}$. Peak blood pressure was normalized to baseline levels and plotted as the mean \pm SD for all groups. Normalization was performed to remove confounding factors such as interanimal variation in baseline parameters. A D'Agostino-Pearson normality test was used, then stimulus response curve data were compared using a two-way ANOVA for repeated-measures followed by Bonferroni's multiple-comparisons test, while a Student's two-tailed paired/unpaired $t$ test was performed to compare the maximal reduction in respiratory rate $\left(\mathrm{E}_{\max }\right)$ and the electrical frequency required to elicit $50 \%$ of the maximum response $\left(\mathrm{EF}_{50}\right)$.

Conscious respiratory studies. Respiratory frequency (breaths/min), tidal volume (milliliters per kilogram), minute volume (milliliters per minute), inspiratory and expiratory time (seconds), peak inspiratory and expiratory flow (milliliters per minute), and enhanced pause (Penh, a measure of airflow obstruction) were automatically sampled from the calibrated Buxco flow trace, corrected for animal body weight and ambient chamber temperature, and calculated in real time by the plethysmography software (Buxco Finepointe Software, version 2.1.0.9). Real-time data collected every $2 \mathrm{~s}$ were used to calculate the average per $5 \mathrm{~min}$ for each cohort at baseline ( $5 \mathrm{~min}$ before vehicle nebulization) and during each nebulized challenge ( 5 min nebulization time). Data were normalized to vehicle nebulization values for each rat and then plotted as the mean \pm SD for all groups. Normalization was performed to remove confounding factors such as interanimal variation in baseline parameters. Data were first tested for normality using a D'Agostino-Pearson test, and the response to each dose of capsaicin was compared between groups using a two-way ANOVA for repeated measures followed by Bonferroni's multiple-comparisons test.

Neuroanatomical data. The efficacy of AAV transduction of the $\mathrm{hM} 3 \mathrm{D}(\mathrm{Gq})$ and control vector were first quantified after conscious physiological studies by manually counting the number of mCherry-positive neurons within the entire rostrocaudal extent of the SubM. Counts from both left and right sides were pooled across the two groups and reported as the mean \pm SD at every $100 \mu \mathrm{m}$. Student's two-tailed unpaired $t$ test was used to compare the $\mathrm{hM} 3 \mathrm{D}(\mathrm{Gq})$ and control AAV groups. In addition, tile-scan images at three representative rostrocaudal levels (distance from bregma: $-2.20,-2.80$, and $-3.30 \mathrm{~mm}$ ) were acquired from each $\mathrm{hM} 3 \mathrm{D}(\mathrm{Gq})$-injected rat with every mCherry-positive neuron (using the region of interest tool on the Leica $\mathrm{X}$ acquisition software) transposed to traces of the SubM to demonstrate the spatial specificity of the injection sites (left and right sides were kept separate). To quantify neuronal activation in transduced SubM neurons following CNO administration, the number of neurons expressing mCherry and/or c-Fos were counted at every $200 \mu \mathrm{m}$ in both hM3D(Gq)-injected and control AAV-injected rats. These counts were presented as a percentage of the total number of mCherry and/or c-Fos neurons in each animal.

A similar approach was used for the anterograde transsynaptic tracing experiments with all H129 tdT-positive neurons counted within the entire rostrocaudal extent of the SubM and reported as the mean \pm SD at every $100 \mu \mathrm{m}$. To demonstrate spatial specificity, traces of individual H129 tdT-positive SubM neurons were obtained from the peak level of infection (distance from bregma, $-2.70 \mathrm{~mm}$ ). 


\section{Results}

Pharmacological modulation of the SubM regulates laryngeal-evoked respiratory reflexes via connectivity to the VLO in anesthetized rats

At optimum stimulus intensities, increasing stimulus frequency applied to the laryngeal mucosa evoked a frequency-dependent reduction in respiratory rate as well as an increase in mean ABP in urethane-anesthetized rats (Fig. $1 B, D, G$ ), while no effect was seen on heart rate $[462.6 \pm 36.0$ beats per minute (bpm) at baseline compared with $473.1 \pm 34.0 \mathrm{bpm}$ at the highest stimulus frequency tested]. It must be noted that baseline heart rate was elevated compared with nonanesthetized rats, perhaps reflective of elevated sympathetic tone commonly observed with urethane anesthesia, and this may have masked any observable change in heart rate during stimulation. Evoked responses during electrical stimulation were substantially reduced following unilateral vagotomy (Fig. $1 C^{\prime}$ ) and were absent following bilateral vagotomy confirming the specificity of the stimulus for vagal afferent pathways (Fig. $\left.1 C^{\prime \prime}\right)$. Bilateral microinjection of vehicle ( $0.9 \%$ saline) into the SubM $(N=9)$ did not alter the stimulation frequency-dependent fall in respiratory rate $\left(\mathrm{EF}_{50}\right.$ and $\mathrm{E}_{\text {max }}$ : paired, $t_{(8)}=1.03$, $p=0.332$, and $t_{(8)}=0.595, p=0.569$, respectively; Fig. $\left.1 D\right)$, or blood pressure $\left(\mathrm{EF}_{50}\right.$ and $\mathrm{E}_{\text {max }}$ : paired, $t_{(9)}=0.4771, p=0.6460$, and $t_{(9)}=0.0423, p=0.9674$, respectively; Fig. $\left.1 G\right)$. Bilateral microinjection of 5-HT into the SubM to excite neurons $(N=9)$ had no effect on basal respiratory rate and blood pressure, but significantly attenuated the respiratory response $\left(\mathrm{EF}_{50}\right.$ and $\mathrm{E}_{\text {max }}$ : paired, $t_{(8)}=10.00, p<0.0001$, and $t_{(8)}=8.13, p<0.0001$, respectively; Fig. $1 E$ ) and modestly reduced the blood pressure response associated with laryngeal stimulation $\left(\mathrm{EF}_{50}\right.$ and $\mathrm{E}_{\max }$ : paired, $t_{(8)}=0.7242, p=0.4962$, and $t_{(8)}=2.329, p=0.0587$, respectively; Fig. $1 H$ ). Bilateral microinjection of muscimol to inhibit neurons into the SubM $(N=6)$ also had no effect on basal respiratory rate and blood pressure and did not modify blood pressure responses evoked by laryngeal stimulation $\left(\mathrm{EF}_{50}\right.$ and $\mathrm{E}_{\text {max }}$ : paired, $t_{(6)}=0.2354, p=1.000$, and $t_{(6)}=0.5410, p=0.6261$, respectively; Fig. 1I). Strikingly, muscimol had the opposite effect of 5-HT, significantly augmenting the respiratory response evoked by laryngeal stimulation $\left(\mathrm{EF}_{50}\right.$ and $\mathrm{E}_{\text {max }}$ : paired, $t_{(5)}=$ 3.02, $p=0.029$, and $t_{(5)}=2.71, p=0.042$, respectively; Fig. $\left.1 F\right)$. Post hoc histologic assessment of the injection sites showed that 9 of 14 rats had successfully received bilateral SubM microinjection "hits," whereas injections in the remaining rats were restricted to unilateral hits. Nevertheless, this variance did not appear to have a significant impact on response magnitudes evoked by 5-HT or muscimol (data not shown). In addition, to determine that the physiological responses observed were SubM specific, six rats received microinjections of 5-HT into brain regions surrounding the SubM (Fig. 2 ). This resulted in no significant change to respiratory rate (Fig. 2A-F), blood pressure, and heart rate (data not shown) after 5-HT microinjection.

Microinjection of the anterograde tracer BDA into the SubM location responsive to 5-HT and muscimol above resulted in dense SubM neuron terminal field labeling in the VLO, spanning from bregma +3.20 to $+4.00 \mathrm{~mm}$. Many of the labeled terminals were concentrated in cortical layer 4 , and less so in layer 5 (Fig. $3 A$ ). Furthermore, retrograde tracing from this VLO site using green fluorescent retrobeads identified a large population of neurons in the SubM (Fig. 3B), confirming the neuroanatomical connection. In functional experiments (Fig. 3C), bilateral sham lesions $(N=3)$ or electrolytic lesions of the $\operatorname{VLO}(N=5)$ followed by bilateral microinjection of vehicle into the SubM had no effect on basal respiratory and blood pressure parameters (Fig. 3D,F). However, VLO lesions significantly potentiated the $\mathrm{E}_{\max }$ evoked by laryngeal stimulation, when compared with the sham lesion rats $\left(\mathrm{EF}_{50}\right.$ and $\mathrm{E}_{\text {max }}$ : unpaired, $t_{(6)}=0.562, p=0.594$, and $t_{(6)}=$ $4.875, p=0.0028$, respectively; Fig. $3 D$ ). Although the potentiating effect was not as substantial as that seen following SubM muscimol microinjection (Fig. $1 F$ ), the data are nonetheless consistent with the VLO lesion unmasking a tonic inhibitory influence of the SubM-VLO circuit on respiratory reflex responses evoked by laryngeal stimulation. No effect of VLO lesion on the laryngeal-evoked blood pressure response was observed in this experiment $\left(\mathrm{EF}_{50}\right.$ and $\mathrm{E}_{\text {max }}$ : unpaired, $t_{(8)}=0.2471, p=0.8120$, and $t_{(8)}=1.132, p=0.2947$, respectively; Fig. $3 F$ ). Bilateral microinjection of 5 -HT into the SubM of sham $(N=4)$ or VLO lesioned $(N=6)$ rats did not alter basal respiratory or blood pressure parameters but characteristically reduced the magnitude of laryngeal-evoked respiratory slowing in sham-lesioned rats (Fig. $3 E, G)$. Strikingly, this effect of 5-HT was blocked entirely in rats receiving bilateral VLO lesions $\left(\mathrm{EF}_{50}\right.$ and $\mathrm{E}_{\text {max }}$ : unpaired, $t_{(8)}=$ 9.061, $p<0.0001$, and $t_{(8)}=7.369, p<0.0001$, respectively; Fig. $3 E)$. A small difference was also seen in the blood pressure response associated with laryngeal stimulation between 5-HTtreated sham-lesioned and VLO-lesioned rats, testing significant for the $\mathrm{E}_{\max }$ response $\left(\mathrm{EF}_{50}\right.$ and $\mathrm{E}_{\max }$ : unpaired, $t_{(8)}=0.2939$, $p=0.7787$, and $t_{(8)}=2.651, p=0.0380$, respectively; Fig. $3 G$ ). This finding is entirely consistent with outputs from the SubM to the VLO playing a central role in SubM-mediated regulation of laryngeal respiratory reflexes.

\section{The SubM-VLO circuit regulates nocifensive respiratory reflexes in conscious rats}

Functional targeting of the SubM-VLO circuit in conscious rats was achieved using DREADD (designer receptor exclusively activated by designer drugs) technology introduced using a combinatorial viral vector approach. In conscious, freely behaving rats undergoing whole-body plethysmography, no change in baseline respiratory parameters were observed $30 \mathrm{~min}$ following administration of $\mathrm{CNO}$ between any of the experimental groups (Table $1)$. In naive rats (i.e., not treated with AAV vectors or $\mathrm{CNO}$; $N=6$ ), increasing concentrations of aerosolized capsaicin produced a dose-dependent increase in respiratory rate and tidal volume (respiratory rate, repeated-measures two-way ANOVA: interaction between group and dose, $F_{(4)}=0.21, p=0.9338$; dose effect, $F_{(4)}=3.19, p=0.0207$; group effect, $F_{(1)}=0.51, p=0.4770$; Fig. $4 A$; tidal volume, repeated-measures two-way ANOVA: interaction between group and dose, $F_{(4)}=0.91, p=0.4656$; dose effect, $F_{(4)}=4.83, p=0.0023$; group effect, $F_{(1)}=2.48, p=0.1216$; Fig. $4 D$ ), the former largely driven by a progressive, albeit variable, reduction in inspiratory time (repeated-measures two-way ANOVA: interaction between group and dose, $F_{(4)}=0.72$, $p=0.5792$; dose effect, $F_{(4)}=3.57, p=0.0123$; group effect, $F_{(1)}=$ $16.60, p=0.0002$; Fig. $4 B$ ). No effect was observed on expiratory time (repeated-measures two-way ANOVA: interaction between group and dose, $F_{(4)}=0.50, p=0.7354$; dose effect, $F_{(4)}=0.50$, $p=0.7351$; group effect, $F_{(1)}=1.52, p=0.2231$; Fig. $4 C$ ). Importantly, CNO did not significantly modify any respiratory parameter response evoked by any concentration of inhaled capsaicin in control experiments involving naive rats (Fig. 4, Table 2 ), arguing that CNO has no observable off-target biological activity in this experimental model. Capsaicin inhalation in CNOtreated control AAV rats $(N=9)$ also produced respiratory responses that were largely indistinguishable from those seen in naive control rats, characterized by a dose-dependent increase in 

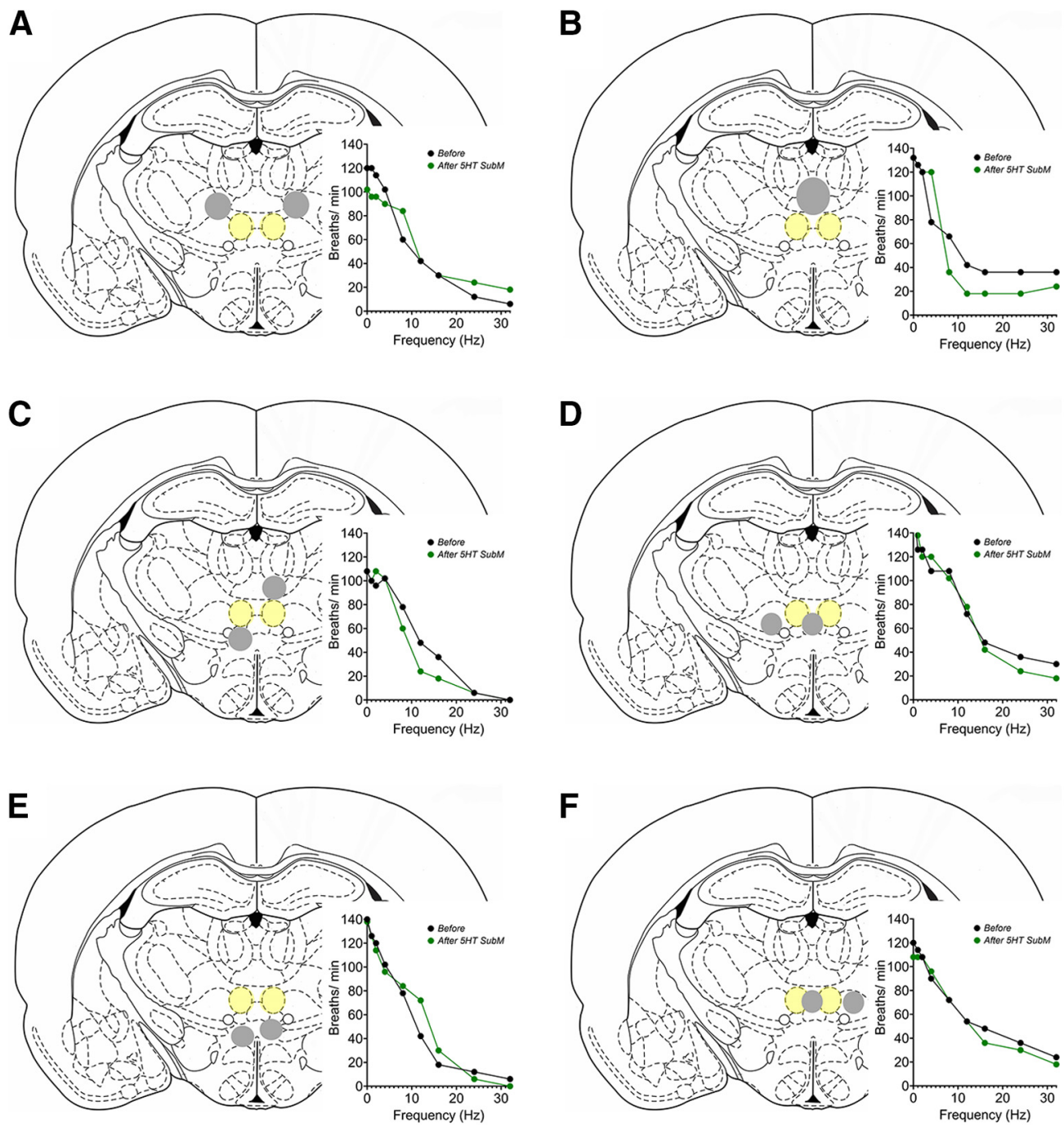

Figure 2. Schematic representations of microinjections of 5-HT into brain regions surrounding the SubM. Each coronal schematic represents an individual rat, with the SubM highlighted in yellow shading and the "missed" injection sites highlighted in gray shading. $\boldsymbol{A}-\boldsymbol{F}$, Microinjection sites encompassed rat 1, ventrolateral and paracentral thalamic nuclei $(\boldsymbol{A})$; rat 2 , central medial thalamic nucleus $(\boldsymbol{B})$; rat 3, central medial, paracentral, and ventral reuniens thalamic nuclei $(\boldsymbol{C})$; rat 4, reuniens and ventromedial thalamic nuclei $(\boldsymbol{D})$; rat 5 , dorsal hypothalamic area $(\boldsymbol{E})$; and rat 6, rhomboid and ventromedial thalamic nuclei $(\boldsymbol{F})$. No significant effect on respiration was observed for each brain region (see graph insert for each brain image). Each coronal slice represents bregma level from -2.70 to $-2.80 \mathrm{~mm}$.

respiratory rate, tidal volume, and a progressive shortening of inspiratory time (Fig. 5C-E, Table 2).

By contrast, CNO administration to rats expressing the $\mathrm{hM} 3 \mathrm{D}(\mathrm{Gq})$ in VLO-projecting SubM neurons $(N=10)$ significantly blunted the dose-dependent capsaicin-evoked increases in respiratory rate (repeated-measures two-way ANOVA: interaction between group and dose, $F_{(4)}=0.24, p=0.917$; dose effect, $F_{(4)}=25.02, p<0.0001$; group effect, $F_{(1)}=40.72, p<0.0001$; Fig. $5 C)$ and the accompanying reduction in inspiratory time (repeated-measures two-way ANOVA: interaction between group and dose, $F_{(4)}=3.58, p=0.011$; dose effect, $F_{(4)}=24.08$, $p<0.0001$; group effect, $F_{(1)}=19.20, p=0.0005$; Fig. $\left.5 D\right)$. Notably, these rats also displayed a significant increase in expiratory time (repeated-measures two-way ANOVA: interaction between group and dose, $F_{(4)}=0.55, p=0.696$; dose effect, $F_{(4)}=$ 5.15, $p=0.0011$; group effect, $F_{(1)}=20.99, p=0.0003$; Fig. $5 E$ ), which resulted in overall respiratory rate falling significantly below baseline levels at capsaicin challenge doses of $0.3-30 \mu \mathrm{M}$ (Fig. 5C). The consistent increase in tidal volume evoked by capsaicin was largely unaffected by $\mathrm{CNO}$ in $\mathrm{hM} 3 \mathrm{D}(\mathrm{Gq})$ rats (repeated-measures two-way ANOVA: interaction between group and dose, $F_{(4)}=2.30, p=0.0674$; dose effect, $F_{(4)}=35.38$, $p<0.0001$; group effect, $F_{(1)}=0.76, p=0.396$; Fig. $\left.5 F\right)$, suggesting that breathing rate and depth are independently regulated by capsaicin inhalation. Overall, the substantial increase in minute ventilation evoked by capsaicin in naive and control AAV-transfected animals was abrogated by the activation of hM3D $(\mathrm{Gq})$ expressed by VLO-projecting SubM neurons and converted to a hypoventilatory response at lower capsaicin challenge concentrations (Table 2). As expected, CNO treatment in these experiments induced the expression of c-Fos in the majority of SubMVLO-projecting neurons virally transfected with $\mathrm{hM} 3 \mathrm{D}(\mathrm{Gq})$ with only a very small number of transfected neurons expressing c-Fos in the control AAV rats (Fig. 5G-I), confirming the specificity of $\mathrm{CNO}$ for hM3D(Gq)-transduced neurons.

The combination of AAVrg pkg-Cre injected into the VLO and AAV8 hSyn-DiO-hM3D(Gq)-mCherry (or control vector) injected into the SubM resulted in robust expression of vector reporter specifically in the SubM neurons with output connectivity to the VLO (Fig. 6A). Transduced neurons spanned a rostrocaudal distribution within the SubM, which ranged from -2.00 to $-3.40 \mathrm{~mm}$ relative to bregma, with the largest density of neurons 
A

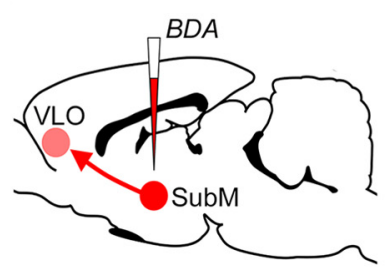

B

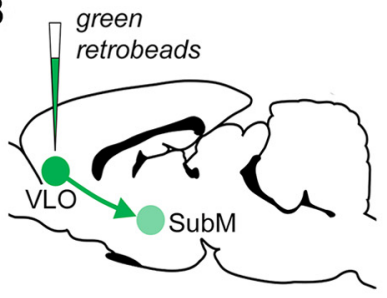

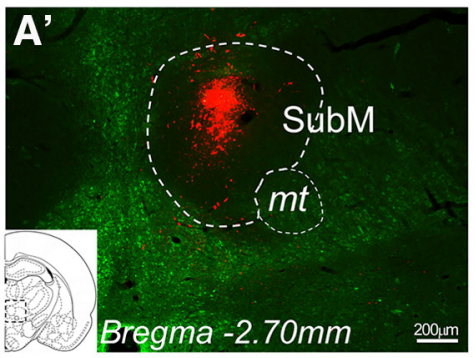

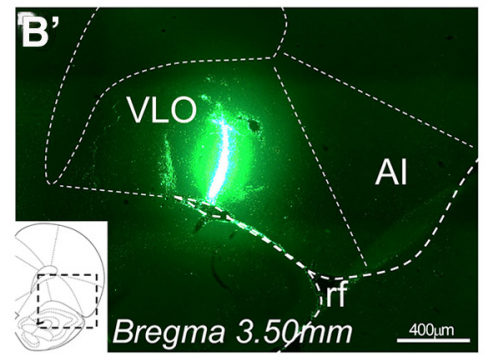

D

C
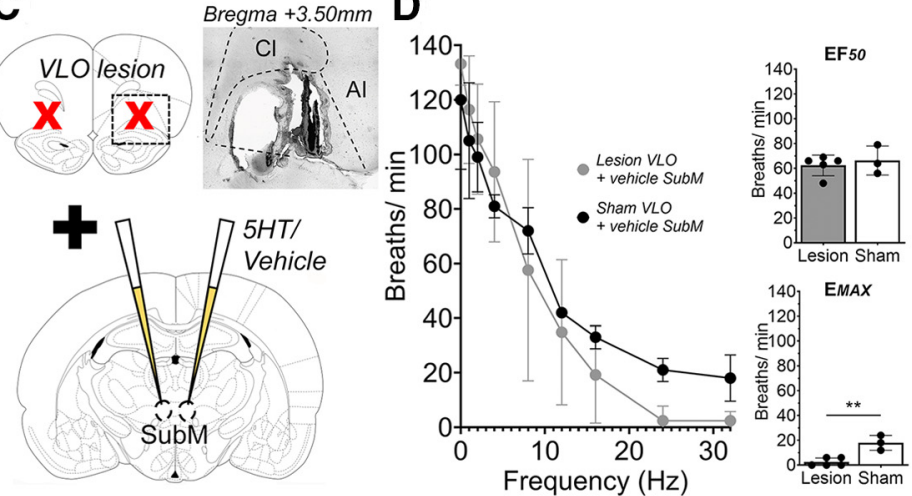

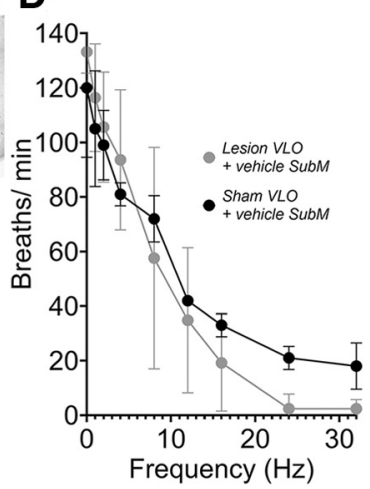

$\mathbf{F}$

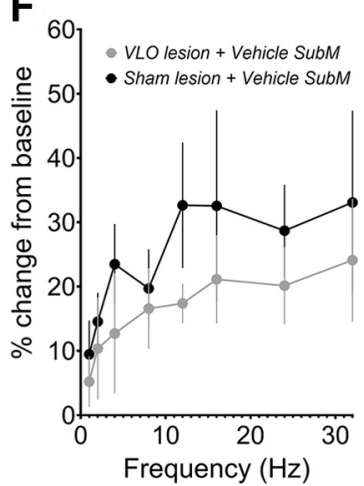

\section{E}
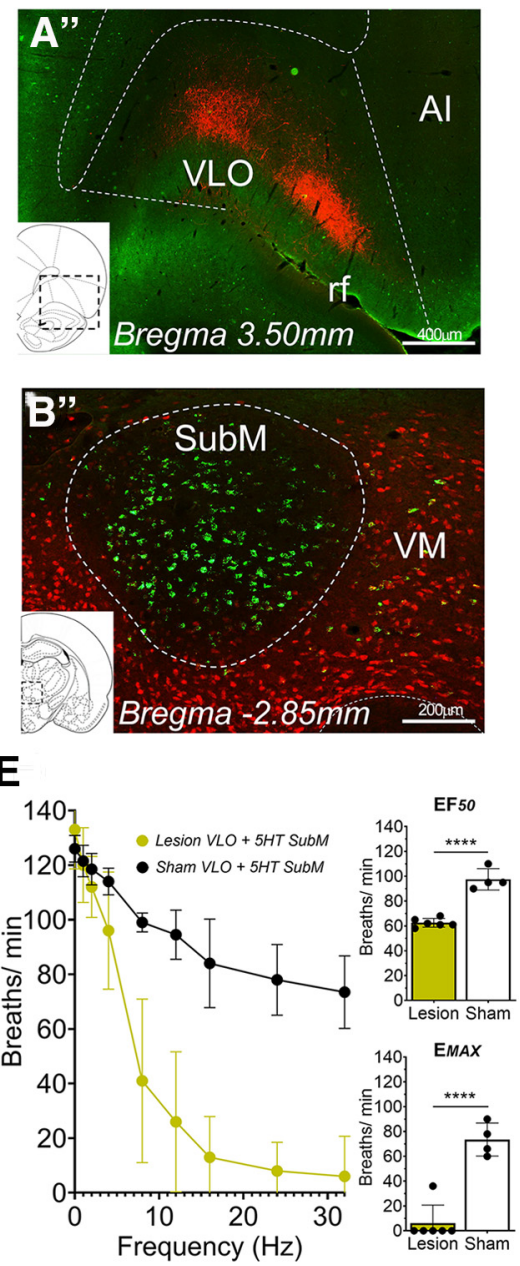

G
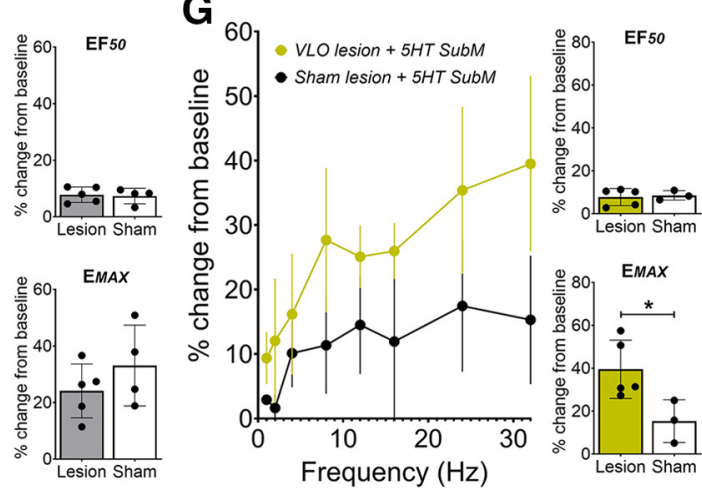

Figure 3. The submedius thalamic nucleus regulates laryngeal-evoked respiratory reflexes in anesthetized rats via connectivity to the ventrolateral orbital cortex. $\boldsymbol{A}, \boldsymbol{B}$, The SubM has dense projections to the VLO, as revealed by anterograde $(\boldsymbol{A})$ and retrograde $(\boldsymbol{B})$ neuroanatomical tracing. $\boldsymbol{C}$, Schematic summary of the physiological experiment design to test the effect of bilateral electrolytic lesioning of the VLO (insert photomicrograph demonstrates example lesion site) or sham lesions on SubM-mediated modulation of laryngeal reflexes. $\boldsymbol{D}$, Laryngeal stimulationevoked changes in respiratory rate for lesion VLO $(N=5)$ and sham VLO $(N=3)$ plus microinjection of vehicle into the SubM. $\boldsymbol{E}$, Laryngeal stimulation-evoked changes in respiratory rate for lesion VLO $(N=6)$ and sham VLO $(N=4)$ plus microinjection of $5-H T$ into the SubM. $F$, Blood pressure stimulus-response curves for lesion VLO $(N=5)$ and sham VLO $(N=3)$ plus microinjection of vehicle into the SubM. $G$, Blood pressure response curves for lesion VLO $(N=6)$ and sham VLO $(N=4)$ plus microinjection of $5-H T$ into the SubM. $* p<0.05, * * p<0.01$, $* * * * p<0.0001$, two-tailed unpaired $t$ test (D-G). Al, Agranular insula; Cl, claustrum; $m t$, mamillary tract; if, rhinal fissure; VM, ventromedial thalamus.

observed at approximately -2.70 to $-2.90 \mathrm{~mm}$. More often these neurons were located within the dorsal half of the nucleus and especially neighboring the dorsal boundary (Fig. $6 B-D$ ). No differences in the spread or positioning of transduced neurons were seen between the hM3D $(\mathrm{Gq})$ and control AAV groups (Fig. 6C). We found evidence for transduced neurons within other brain regions located in central and medial thalamus; however, the number of transduced neurons in each region was significantly lower than that observed in the SubM of $\mathrm{hM} 3 \mathrm{D}(\mathrm{Gq})$ rats (oneway ANOVA: $F_{(8)}=15.87, p<0.0001$; Fig. $6 A$ ).

\section{Specificity of the SubM-VLO circuit for vagal processing via the Pa5}

Airway afferents that terminate in the medullary Pa5 are known to project to the SubM (McGovern et al., 2015a). Using the same anterograde transsynaptic tracing approach with our conditional 
Table 1. Baseline respiratory measurements after CNO administration in naive $(N=6)$, naive $+3 \mathrm{mg} / \mathrm{kg}$ CNO $(N=6)$, control AAV $+3 \mathrm{mg} / \mathrm{kg}$ CNO (N=9), and $\mathrm{hM} 3 \mathrm{D}(\mathrm{Gq})+3 \mathrm{mg} / \mathrm{kg}$ CNO rats $(N=10)$ rats

\begin{tabular}{lcccc}
\hline & Naive $(N=6)$ & Naive $+3 \mathrm{mg} / \mathrm{kg}$ CNO $(N=6)$ & Control AAV + 3 mg/kg CN0 (N=9) & $\mathrm{hM3D}(\mathrm{Gq})+3 \mathrm{mg} / \mathrm{kg}$ CN0 (N= 10) \\
\hline Respiratory rate (breaths/min) & $100.1 \pm 6.89$ & $93.23 \pm 9.63$ & $95.56 \pm 7.18$ & $99.92 \pm 11.24$ \\
Inspiratory time (s) & $0.24 \pm 0.03$ & $0.29 \pm 0.03$ & $0.26 \pm 0.03$ & $0.25 \pm 0.05$ \\
Expiratory time (s) & $0.35 \pm 0.03$ & $0.38 \pm 0.05$ & $0.39 \pm 0.03$ & $0.39 \pm 0.05$ \\
Tidal volume (ml/breath) & $1.81 \pm 0.28$ & $2.03 \pm 0.43$ & $1.97 \pm 0.20$ & $2.13 \pm 0.37$ \\
Minute volume (ml/min) & $178.3 \pm 34.35$ & $185.30 \pm 30.57$ & $184.60 \pm 24.67$ & $208.80 \pm 51.34$ \\
Peak inspiratory flow rate (ml/min) & $10.19 \pm 3.80$ & $11.77 \pm 2.51$ & $14.14 \pm 2.69$ & $14.96 \pm 3.83$ \\
Peak expiratory flow rate (ml/min) & $8.10 \pm 1.84$ & $9.59 \pm 1.46$ & $9.43 \pm 0.98$ & $10.35 \pm 2.01$ \\
Penh & $1.16 \pm 0.34$ & $1.00 \pm 0.16$ & $0.85 \pm 0.17$ & $1.00 \pm 0.35$ \\
\hline
\end{tabular}

Measurements were taken $30 \mathrm{~min}$ after intraperitoneal CNO administration and represent the average $5 \mathrm{~min}$ of recording before the first nebulization (vehicle). Data are represented as the mean \pm SD for each group, and no significant differences were found between groups as determined by one-way ANOVA.
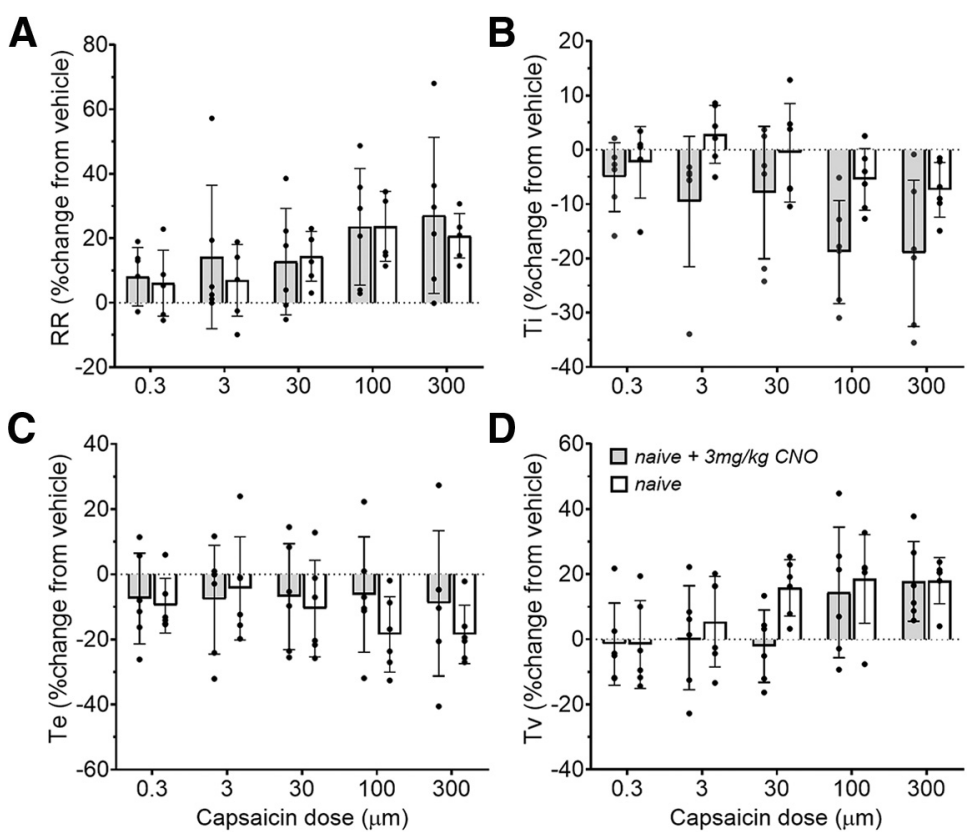

Figure 4. Respiratory parameters measured during conscious capsaicin challenge are unaltered by CNO in the absence of $\mathrm{hM} 3 \mathrm{D}(\mathrm{Gq})$ DREADD expression. $\boldsymbol{A}-\boldsymbol{D}$, Respiratory rate $(\mathrm{RR} ; \boldsymbol{A})$, inspiratory time $(\mathrm{Ti} ; \boldsymbol{B})$, expiratory time (Te; $\boldsymbol{C}$, and tidal volume (Tv; $\boldsymbol{D})$ in naive $(N=6)$ or naive $+3 \mathrm{mg} / \mathrm{kg}$ CNO $(N=6)$ rats

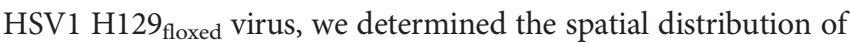
H129 tdT-infected neurons within the SubM that are in receipt of Pa5-projecting airway afferents. Infected neurons spanned a rostrocaudal distribution within the SubM that ranged from -2.30 to $-3.20 \mathrm{~mm}$, with the largest density observed at approximately $-2.70 \mathrm{~mm}$ (Fig. $6 \mathrm{G}$ ). Interestingly, the majority of these neurons was located in the dorsal half of the SubM nucleus (Fig. $6 G, H)$, consistent with what we observed with the transduction patterns of both the $\mathrm{hM} 3 \mathrm{D}(\mathrm{Gq})$ and control AAV experiments. Activation of vagal afferent central terminals in both the NTS and $\mathrm{Pa} 5$ was achieved by microinjecting capsaicin into these two brain sites. Following SubM treatment with vehicle, microinjection of capsaicin $(0.5 \mu \mathrm{M})$ into the medullary caudal NTS $(N=8$; Fig. $7 A$ ) produced a considerable decrease in respiratory frequency (Fig. $7 B, C$ ) and a decrease in blood pressure (Fig. 7B,D). No change was observed in these parameters following activation of the SubM with 5-HT (repeated-measures two-way ANOVA: interaction between time and treatment, $F_{(10)}=0.51, p=0.8723$; time effect, $F_{(10)}=10.64, p<0.0001$; treatment effect, $F_{(1)}=0.08$, $p=0.7881$; Fig. $7 C, D$ ). Microinjection of capsaicin into the Pa5 $(N=8$; Fig. $7 E)$ following SubM vehicle treatment decreased respiratory rate (Fig. $7 F, G$ ) and produced a substantial increase in blood pressure (Fig. 7F,H). Activation of the SubM with 5-HT was able to block the decrease in respiratory rate (repeated-measures two-way ANOVA: interaction between time and treatment, $F_{(10)}=5.56, p<0.0001$; time effect, $F_{(10)}=$ $1.11, p=0.3691$; treatment effect, $F_{(1)}=208.57$, $p<0.0001$; Fig. $7 G$ ) observed following capsaicin microinjection into the Pa5; however, no effect was seen in blood pressure (Fig. $7 H$ ).

\section{Discussion}

We provide compelling evidence that vagal airway nociceptive inputs to the brainstem are regulated by a central descending control system, capable of substantially blunting airway nociceptor reflexes. Essential to this system is circuitry between the SubM and VLO, confirmed in the present study using a combination of conventional and viral neuroanatomical tracing, and conventional and chemogenetic functional studies. Descending inhibition of nociceptive sensory processing orchestrated by the SubM-VLO circuit was demonstrated at the level of vagal sensory processing sites within the brainstem $\mathrm{Pa} 5$, where jugular afferents terminate, but not in the caudal NTS, where nodose afferents project. These observations resemble the thalamocortical network regulating the endogenous analgesia system, which exerts comparable regulation of spinal dorsal horn nociceptive processing (Tang et al., 2009). The potential intersection of the endogenous analgesia system with jugular vagal sensory processing in the brainstem offers new insight into the regulation of airway nociceptive reflexes and respiratory sensations.

\section{A SubM-VLO circuit provides both tonic and recruitable top-down control over vagal somatosensory processing}

The somatosensory innervation of the nose and pharynx (supplied by trigeminal and glossopharyngeal nerves) is partially preserved in the larynx and proximal airways via innervation from the jugular vagal ganglia, before transitioning to predominantly viscerosensory nodose innervation throughout the remainder of the airways and lungs (McGovern et al., 2015b; Mazzone and Undem, 2016). A somatosensory representation of the airways in the brain has important functional implications, including for the perception of airway irritation. For example, studies of noxious spinal processing have shown that although graded sensory stimuli generally result in graded action potential responses in dorsal horn neurons, the level of sensation experienced may not 
Table 2. Respiratory parameters measured during conscious capsaicin challenge in in naive $(N=6)$, naive $+3 \mathrm{mg} / \mathrm{kg}$ CNO ( $N=6)$, control AAV $+3 \mathrm{mg} / \mathrm{kg}$ CNO $(N=9)$, and $\mathrm{hM} 3 \mathrm{D}(\mathrm{Gq})+3 \mathrm{mg} / \mathrm{kg}$ CNO rats $(N=10)$ rats

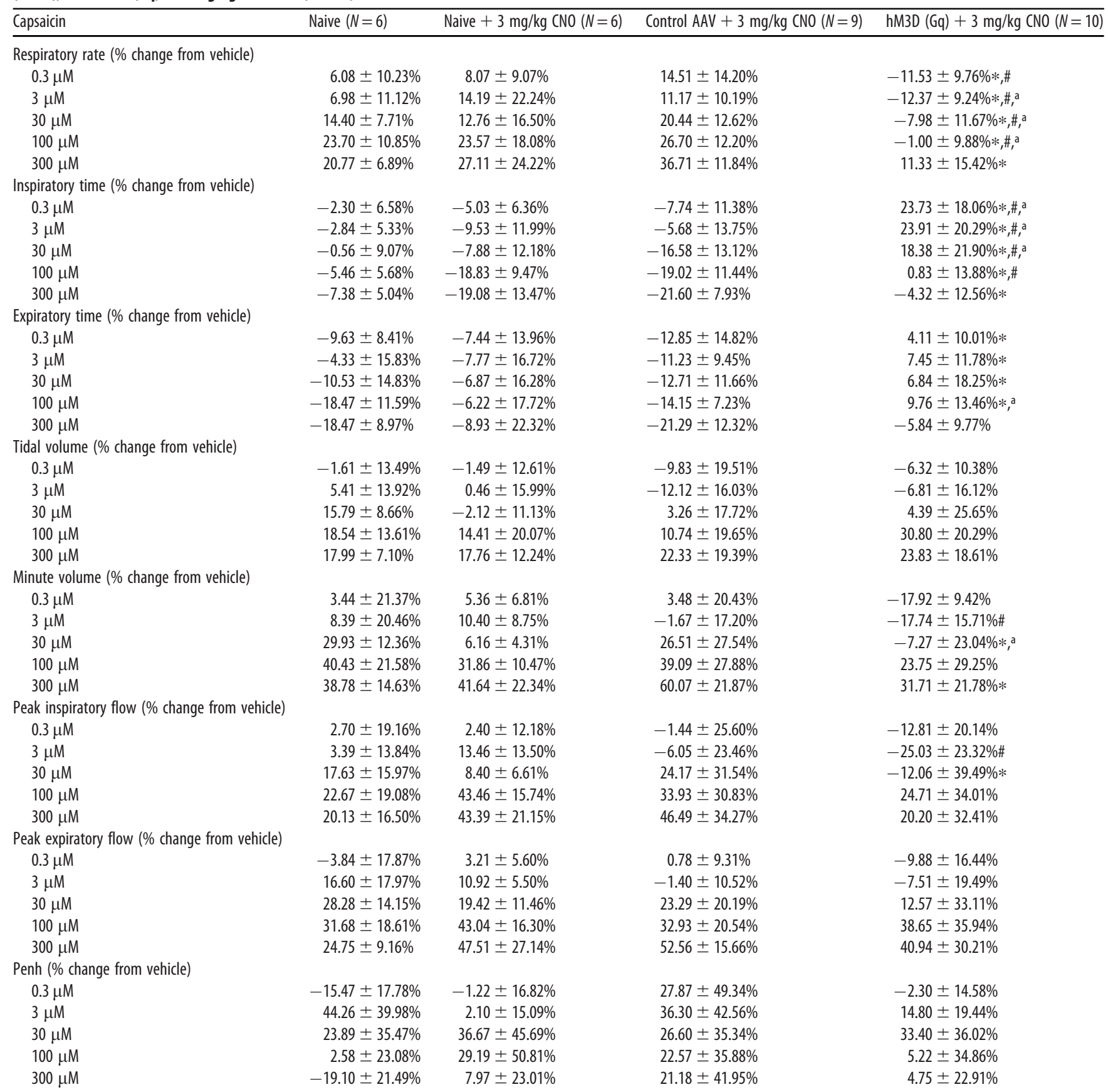

Data are represented as the mean \pm SD for each group challenged with increasing doses of capsaicin. $*, \#$, ahM3D(Gq) + CNO group significantly different versus control AAV + CNO, naive + CNO, and naive groups, respectively, as determined by Bonferroni's multiple-comparisons test.

*,\#,ahM3D(Gq) + CNO group significantly different to Control AAV + CNO group, naive + CNO group, or naive group, respectively as determined by Bonferroni's multiple-comparisons test.

correlate with these measures as "top-down" neural pathways are capable of modulating nociceptive processing in the spinal dorsal horn (Millan, 2002; Schweinhardt and Bushnell, 2010; Ossipov et al., 2014). One prominent pathway involves descending projections from the prefrontal cortex (e.g., rostral agranular insular cortex and VLO) to the midbrain periaqueductal gray (PAG), a region central to the endogenous analgesia system. Indeed, stimulation of these prefrontal cortex regions in animals, or the dorsolateral prefrontal cortex in humans, induces analgesia (Hardy, 1975; Lee et al., 2015; Cheriyan and Sheets, 2018; Ong et al., 2019; Wu et al., 2020). Although a wide variety of inputs can regulate prefrontal cortical activity, inputs from the SubM have been shown to be a major contributor to prefrontal-mediated analgesia (Zhang et al., 1998; Tang et al., 2009). The present study suggests that this same network regulates aspects of vagal sensory processing in the brainstem.

We used reflex measures of breathing and autonomic tone as indicators of central vagal afferent circuit activity. As previously demonstrated (Driessen et al., 2015, 2020), electrical stimulation of the larynx evoked a vagally mediated cardiorespiratory response that mimicked the laryngeal chemoreflex. Our data confirm previous reports of dense neuroanatomical connectivity between the SubM and VLO (Coffield et al., 1992; Yoshida et al., 1992; Kuramoto et al., 

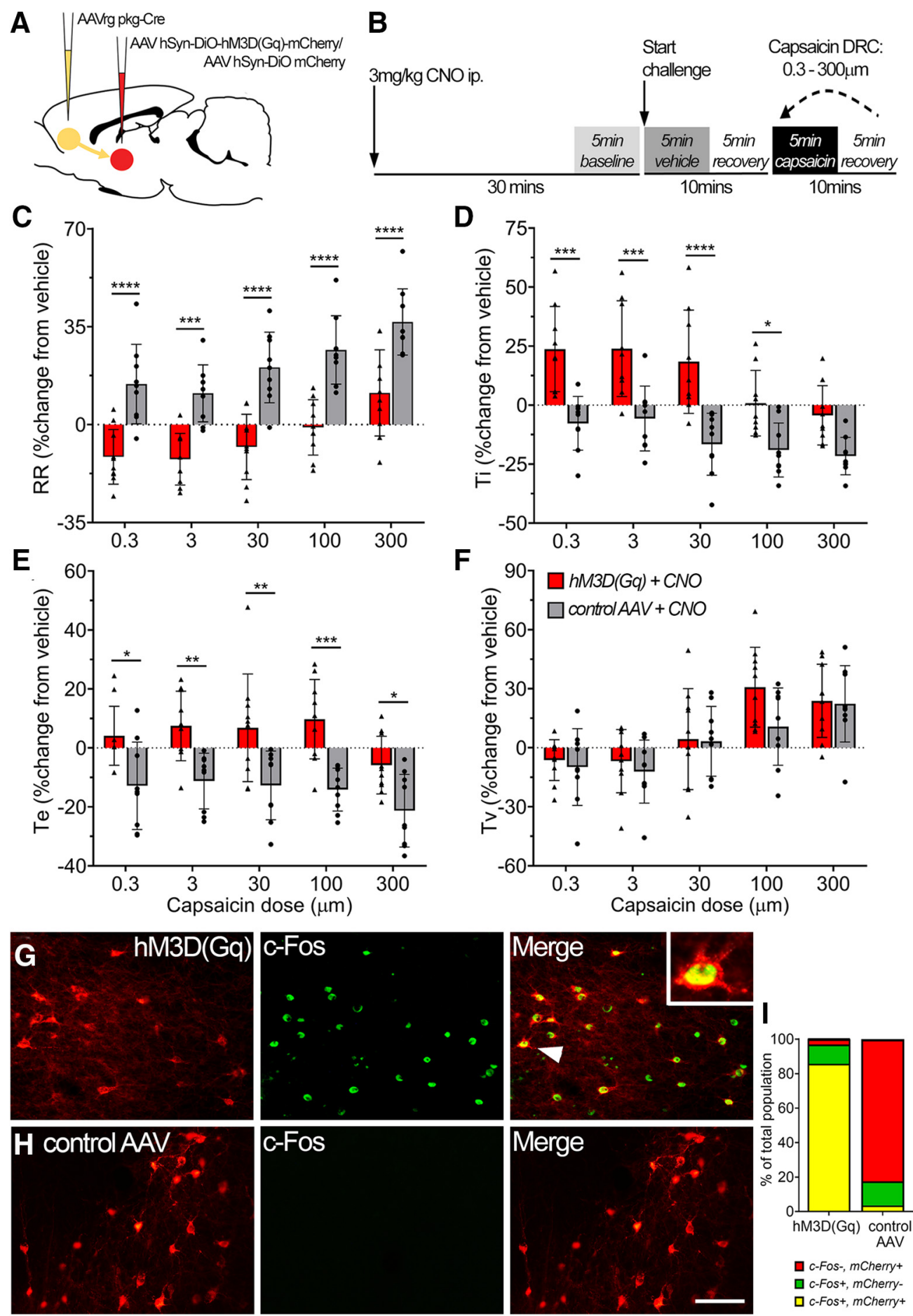

Figure 5. Activation of the SubM-VLO circuit in conscious unrestrained rats regulates nocifensive respiratory reflexes induced by inhalation of capsaicin. $A$, Microinjection of AAVrg pkg-Cre into the VL0 to achieve Cre-dependent expression of either hM3D(Gq) mCherry or control mCherry in SubM-VL0 projecting neurons only. $\boldsymbol{B}$, Schematic outlining the conscious respiratory challenge protocol. $\boldsymbol{C}-\boldsymbol{F}$, Respiratory parameters measured during conscious capsaicin challenge in $\mathrm{hM} 3 \mathrm{D}(\mathrm{Gq})+\mathrm{CNO}(N=10)$ or control AAV + CNO $(N=9)$ rats: respiratory rate (RR; $\boldsymbol{C}$ ), inspiratory time (Ti; $\boldsymbol{D}$ ), expiratory time (Te; $\boldsymbol{E}$ ), Tidal volume (Tv; $\boldsymbol{F})$. $\boldsymbol{G}, \boldsymbol{H}$, Representative images of c-Fos (green) expression in SubM neurons expressing either $\mathrm{hM} 3 \mathrm{D}(\mathrm{Gq}) \mathrm{mCherry}(\boldsymbol{G})$ or control AAV mCherry $(\boldsymbol{H})$ following administration of $\mathrm{CNO} 2 \mathrm{~h}$ before rats were killed. Arrowhead indicates colabeled neuron in box insert. $\boldsymbol{I}$, Quantification of the number of SubM neurons expressing $\mathrm{mCherry}$ and/or c-Fos represented as a percentage of the total number of $\mathrm{mCherry}$ and/or c-Fos neurons counted $[\mathrm{hM} 3 \mathrm{D}(\mathrm{Gq}), 529$ neurons; control AAV, 580 neurons]. $* p<0.05$; $* * p<0.01 ; * * * p<0.001 ; * * * * p<0.0001, \mathrm{hM} 3 \mathrm{D}(\mathrm{Gq})$ versus control AAV by Bonferroni's multiple comparisons (C-F). Scale bars: $\mathbf{G}, \boldsymbol{H}, 75 \mu \mathrm{m}$.

2017). When activated by 5-HT in anesthetized rats, this SubM-VLO circuit produced potent inhibition of breathing (and to a lesser extent cardiovascular) reflexes evoked by directly stimulating the larynx. This functional effect is unlikely to be an artifact unmasked in anesthetized animals as targeted chemogenetic stimulation of the SubM-VLO circuit in conscious rats potently inhibited respiratory responses evoked by inhaling the nociceptive agent capsaicin.

Two observations from our physiological studies are of particular interest. First, no intervention performed in the SubM or VLO modified basal respiratory or cardiovascular measures, suggesting that SubM-VLO circuitry is devoid of regulatory inputs 
A

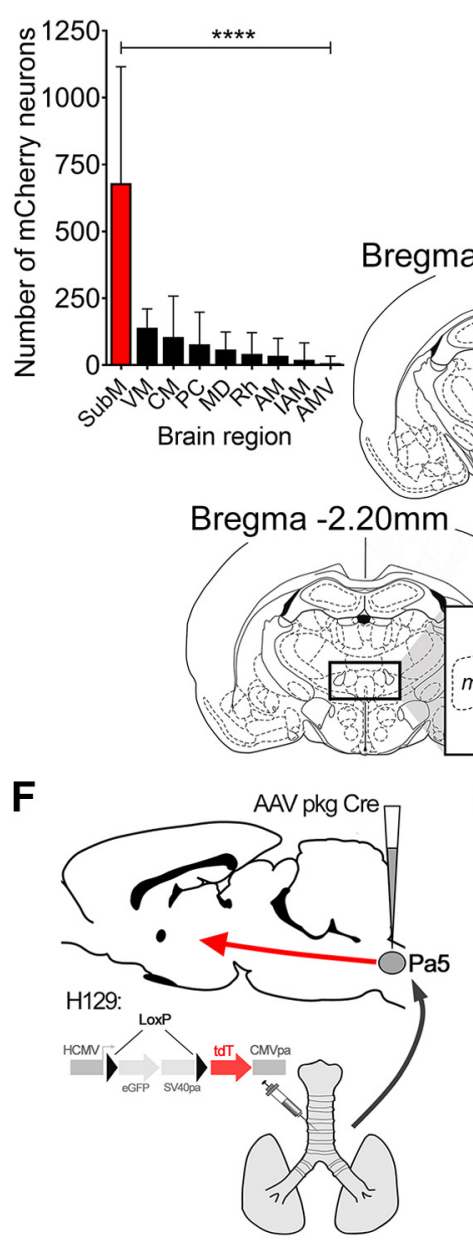

B

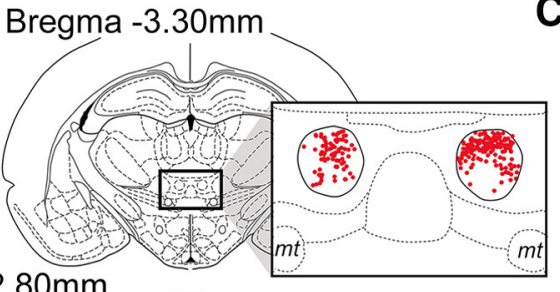

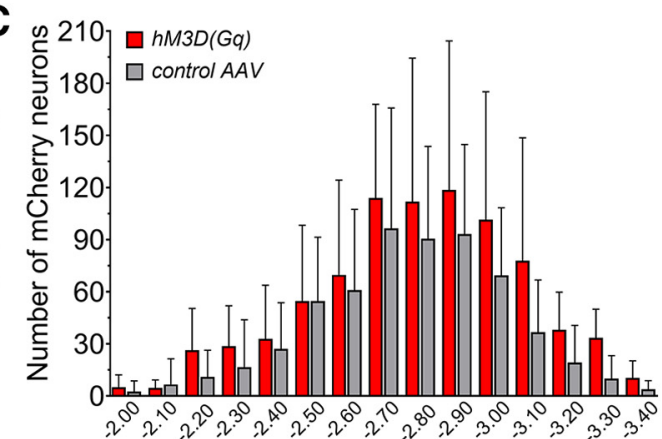

Bregma level (mm)
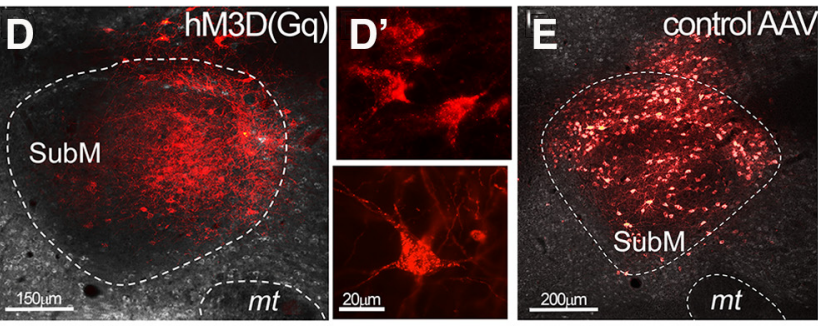

G

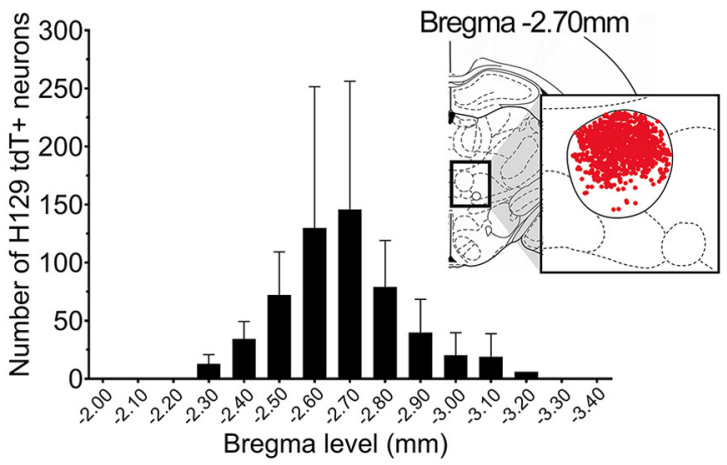

Figure 6. Neuroanatomical organization of neurons in the submedius thalamic nucleus involved in modulating vagal reflexes. $\boldsymbol{A}$, Total number of transduced neurons in the brain of hM3D $(\mathrm{Gq}) \mathrm{mCherry}$ rats $(N=10)$. Data are presented as the mean \pm SD for each brain region. $\boldsymbol{B}$, Schematic representation of the distribution of VL0-projecting hM3D(Gq)-transduced neurons within the SubM. Each coronal slice summarizes the location of individual hM3D(Gq) mCherry neurons (red dots, from all hM3D(Gq) rats, $N=10)$ at three different bregma levels spanning the entire rostrocaudal extent of the SubM. C, Quantification of the rostrocaudal spread (at $100 \mu \mathrm{m}$ intervals) of neurons transduced by hM3D(Gq) and control AAV ( $N=9$ rats). Data are represented as the mean \pm SD. $\boldsymbol{D}-\boldsymbol{E}$, Representative images of hM3D(Gq) mCherry-transduced neurons $(\boldsymbol{D})$ with examples of high-power individual neurons $\left(\boldsymbol{D}^{\prime}\right)$ and control AAV mCherry-transduced neu-

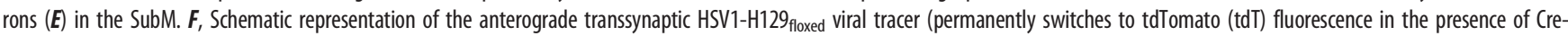
recombinase; McGovern et al., 2015a) injected into the airways of rats that had previously been microinjected with the AAV pkg (re construct into the medullary Pa5. G, Rostrocaudal spread of SubM H129 tdT-infected neurons in receipt of Pa5 projecting airway neurons at $100 \mu$ m intervals $(N=6)$. Data are represented as the mean \pm SD. Coronal slice summarizes the location of individual tdT neurons (red dots, from all rats) at the peak bregma level of $\mathrm{H} 129 \mathrm{tdT}$ infection. $\boldsymbol{H}$, Representative image of $\mathrm{H} 129 \mathrm{tdT}$-infected neurons in the SubM. $* * * * p<0.0001$, SubM, determined by Bonferroni's multiple comparisons $(\boldsymbol{A})$. VM, Ventromedial thalamic nucleus; $\mathrm{CM}$, central medial thalamic nucleus; PC, paracentral thalamic nucleus; MD, mediodorsal thalamic nucleus; Rh, rhomboid thalamic nucleus; AM, anteromedial thalamic nucleus; IAM, interanteromedial thalamic nucleus; AMV, anteromedial ventral thalamic nucleus; mt, mamillary tract.

directly to cardiorespiratory premotor/motor neuron pools in the brainstem and spinal cord. The data may also argue that ongoing pulmonary stretch receptor feedback (nodose derived) to the brainstem is also not regulated by SubM-VLO activity, as alterations in this feedback would be expected to modify basal breathing (Nonomura et al., 2017). However, an action mediated at these sites cannot be categorially ruled out without detailed electrophysiological examination. Second, both SubM inhibition by muscimol and VLO lesion demonstrated a level of SubMVLO circuit-dependent inhibitory tone over vagal sensory processing in the brainstem. This observation argues for the existence of central neuronal mechanisms capable of bidirectionally altering the gain of laryngeal-evoked respiratory reflexes.

Precisely how the SubM-VLO circuit induces the inhibition of vagal nociceptive respiratory responses is unclear. In spinal nociceptive processing, prefrontal neurons descend on PAG neurons, which in turn regulate spinally projecting brainstem neuronal pools in the rostral ventromedial medulla, locus coeruleus, and previously (Millan, 2002; Kim et al., 2018). A similar circuit (or components of it) may be involved in vagal sensory regulation in the brainstem. As we have previously reported, respiratory reflexes can be evoked by microinjections of capsaicin into either the NTS or Pa5, responses that are likely mediated in part by airway vagal afferents terminating in these regions (Mazzone and Canning, 2002; Kubin et al., 2006; Chang et al., 2015; Kim et al., 2020). Concomitant activation of the SubM significantly blunted capsaicin-evoked responses from the Pa5, without having an effect on those mediated by the NTS. This strongly indicates that SubM-VLO descending inhibitory influences are restricted to jugular somatosensory vagal afferent pathways. This observation is perhaps consistent with the disproportionate impact on breathing reflexes over the accompanying cardiovascular responses, of which the latter are dominated by the activation nodose and NTS circuits (Kalia and Richter, 1988; Bonham and 
A

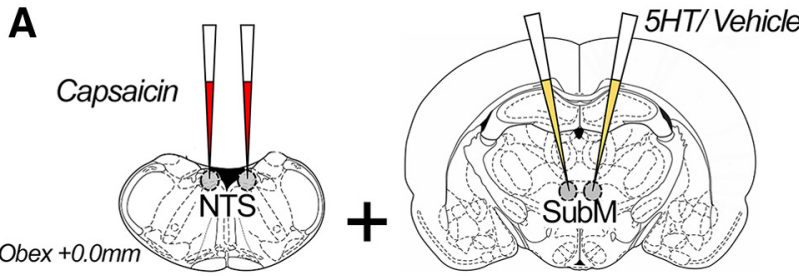

B Respiration
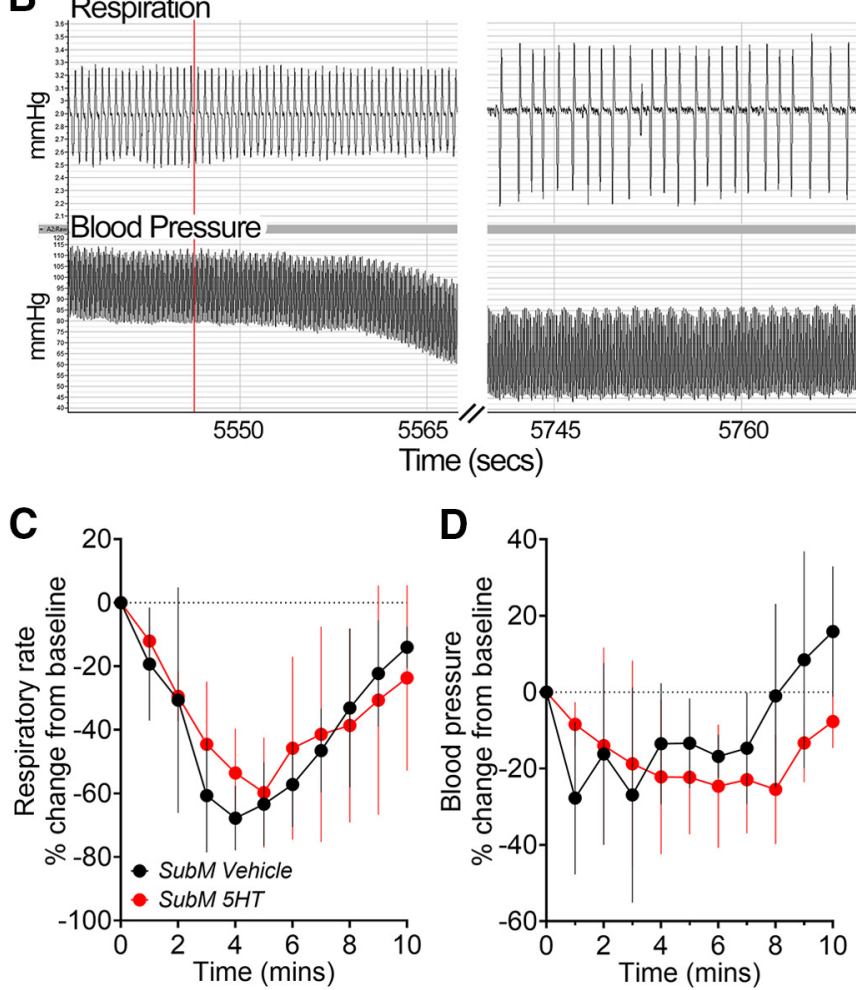
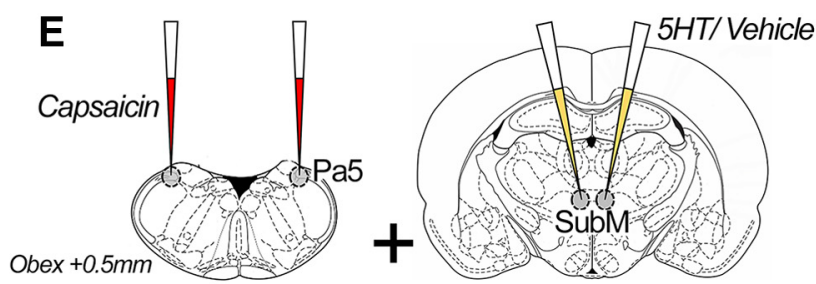

F Respiration
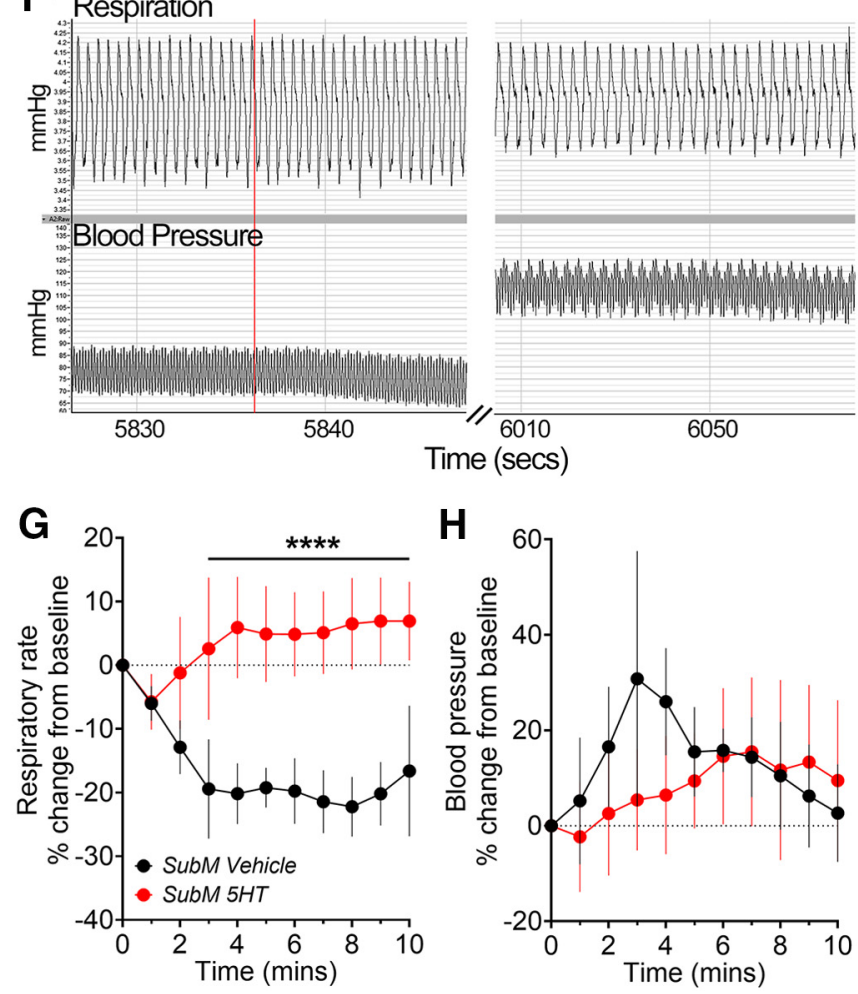

Figure 7. Differential effects on stimulation of bulbar vagal integration nuclei mediated by the submedius thalamic nucleus. $\boldsymbol{A}, \boldsymbol{E}$, Schematic of experimental design showing bilateral microinjections of vehicle or 5 -HT into the SubM, preceding microinjection of capsaicin into either the Pa5 $(\boldsymbol{N}=8)$ or NTS $(\boldsymbol{N}=8)$ to activate the central terminals of primary nociceptors. $\boldsymbol{B}-\boldsymbol{D}, \boldsymbol{F}-\boldsymbol{H}$, Representative chart traces show respiratory and blood pressure movements before and after bilateral microinjection of capsaicin (red line) into either the NTS $(\boldsymbol{B})$ or Pa5 ( $\boldsymbol{F}$ ) following vehicle microinjection into the SubM. NTS capsaicin-evoked changes in respiratory frequency $(\boldsymbol{C})$ and blood pressure $(\boldsymbol{D})$ were unaffected, whereas Pa5 capsaicin-evoked changes in respiratory frequency $(\boldsymbol{G})$, but not blood pressure $(\boldsymbol{H})$, were significantly blunted by SubM 5-HT microinjection in anesthetized rats. Data are represented as the mean \pm SD. $* * * * p<0.0001$, SubM 5-HT versus SubM vehicle by Bonferroni's multiple-comparisons test $(\boldsymbol{B})$.

McCrimmon, 1990; Kubin et al., 2006; Mazzone and Undem, 2016). Furthermore, in conscious rats, capsaicin inhalation evoked a paradoxical hypoventilation after activation of the SubM-VLO circuit, rather than simply preventing the increase in breathing observed in control animals, conceivably representing an underlying or alternate reflex response not sensitive to SubM-VLO activation.

\section{Vagal inputs may regulate thalamocortical control of the descending analgesia system}

The SubM contains glutamatergic neurons that project to the VLO and local interneurons responsive to noxious stimuli from a range of peripheral tissues (Zhang et al., 1998; Huo et al., 2009; Kuramoto et al., 2017). Previous studies have shown that it receives direct nociceptive inputs from laminar one neurons of the trigeminal nucleus and spinal dorsal horn (Craig and Burton, 1981; Ma et al., 1988; Dado and Giesler, 1990; Yoshida et al., 1991; Blomqvist et al., 1992). Using novel transsynaptic herpes viral tracing strategies, we confirm that airway vagal afferent pathways reach the SubM and VLO, terminating in regions that overlap extensively with other defined nociceptive inputs
(McGovern et al., 2012, 2015a,b). The anatomic route of these vagal inputs is solely via the $\mathrm{Pa} 5$, indicative of only afferents of jugular vagal ganglia origin contributing to nociceptive regulation of the SubM-VLO circuit. Indeed, we previously reported that projections into the SubM and VLO are strikingly absent from nodose-NTS circuits (McGovern et al., 2015a). Visceral inputs via spinal routes to this thalamocortical circuit are well described. For example, both colorectal distension and noxious somatic stimulation have been shown to modulate the activity of the same neurons in the SubM and VLO (Kawakita et al., 1993, 1997; Fu et al., 2002; Barkova et al., 2005). Our data suggest that an alternative input from internal organs may arise from tissues supplied by the jugular vagal ganglia. The circumstances under which vagal input to the SubM-VLO circuit is recruited is not known. Conceivably, as the cervical vagus nerve and especially its proximal branches (e.g., the auricular nerve) contains axons arising from jugular ganglia neurons, our anatomic and functional data linking the jugular sensory pathway with the endogenous analgesia system offers an alternative circuitry via which vagus nerve stimulation protocols modulate pain and spinal nociceptive transmission (Randich and Gebhart, 1992; Ren et al., 
1993; Multon and Schoenen, 2005; Straube et al., 2015; Mahadi et al., 2019).

\section{Conclusions and implications for disorders of airway sensory processing}

Our results further support the notion that airway tissues have a vagal somatosensory representation in the brain. Our extensive past and present animal and human data argue that this central somatosensory circuitry arises from jugular vagal afferents and involves relays via the Pa5 and ventrobasal thalamus (McGovern et al., 2015a,b; Farrell et al., 2020). Cough is a significant and difficult to treat clinical problem, often ascribed to disordered functioning of nociceptive vagal afferents of the nodose ganglia. However, mounting evidence is challenging this dogma. Animal and human studies support the notion that jugular, not nodose vagal nociceptive, pathways evoke coughing when stimulated (Driessen et al., 2016; Hewitt et al., 2016; Chou et al., 2018; Driessen et al., 2020). Intriguingly, both behavioral studies and clinical trials of new cough suppressants show profound inhibitory effects of placebo conditioning (up to $40 \%$ reduction) on cough and measures of airway irritant perception (Abdulqawi et al., 2015; Morice et al., 2019). We have shown in humans that placebo cough suppression is proportional to enhanced prefrontal cortex activity (Leech et al., 2013), in regions also involved in placebo analgesia and the recruitment of the endogenous analgesia system. When considered alongside the present data, these observations point toward a clinically relevant role of jugular vagal afferent pathways and descending control mechanisms in chronic cough. Given that altered central inhibition is common in patients with chronic pain, it will be intriguing to understand how this system functions in disorders impacting the airways. Chronic cough patients and long-term cigarette smokers both show signs of altered central inhibition, significant changes in PAG activity and cough control (Ando et al., 2016, 2019), suggesting that therapies aimed at reducing jugular vagal sensory processing or enhancing central inhibition may prove clinically useful for treating excessive coughing. Our findings may equally offer new insights into other pathophysiological conditions, such as migraine and nociceptive hypersensitivities in head, neck, and thoracic tissues known to receive jugular vagal afferent innervation.

\section{References}

Abdulqawi R, Dockry R, Holt K, Layton G, McCarthy BG, Ford AP, Smith JA (2015) P2X3 receptor antagonist (AF-219) in refractory chronic cough: a randomised, double-blind, placebo-controlled phase 2 study. Lancet 385:1198-1205.

Abubakar AB, Bautista TG, Dimmock MR, Mazzone SB, Farrell MJ (2020) Behavioral and regional brain responses to inhalation of capsaicin modified by painful conditioning in humans. Chest. S0012-3692(20)34437-8.

Ando A, Smallwood D, McMahon M, Irving L, Mazzone SB, Farrell MJ (2016) Neural correlates of cough hypersensitivity in humans: evidence for central sensitisation and dysfunctional inhibitory control. Thorax 71:323-329.

Ando A, Mazzone SB, Farrell MJ (2019) Altered neural activity in brain cough suppression networks in cigarette smokers. Eur Respir J 54:1900362.

Barkova E, Turnbull GK, Downie JW (2005) Colonic nociception via nucleus submedius is modulated by pontine centres in the rat. Neurosci Lett 384:193-197. 19

Basbaum AI, Fields HL (1984) Endogenous pain control systems: brainstem spinal pathways and endorphin circuitry. Annu Rev Neurosci 7:309-338.

Bautista TG, Leech J, Mazzone SB, Farrell MJ (2019) Regional brain stem activations during capsaicin inhalation using functional magnetic resonance imaging in humans. J Neurophysiol 121:1171-1182.
Bonham AC, McCrimmon DR (1990) Neurones in a discrete region of the nucleus tractus solitarius are required for the Breuer-Hering reflex in rat. J Physiol 427:261-280.

Blomqvist A, Ericson AC, Broman J, Craig AD (1992) Electron microscopic identification of lamina I axon terminations in the nucleus submedius of the cat thalamus. Brain Res 585:425-430.

Chang RB, Strochlic DE, Williams EK, Umans BD, Liberles SD (2015) Vagal sensory neuron subtypes that differentially control breathing. Cell 161:622-633.

Cheriyan J, Sheets PL (2018) Altered excitability and local connectivity of mPFC-PAG neurons in a mouse model of neuropathic pain. J Neurosci 38:4829-4839.

Chou YL, Mori N, Canning BJ (2018) Opposing effects of bronchopulmonary C-fiber subtypes on cough in guinea pigs. Am J Physiol Regul Integr Comp Physiol 314:R489-R498.

Coffield JA, Bowen KK, Miletic V (1992) Retrograde tracing of projections between the nucleus submedius, the ventrolateral orbital cortex, and the midbrain in the rat. J Comp Neurol 321:488-499.

Craig AD, Burton H (1981) Spinal and medullary lamina I projection to nucleus submedius in medial thalamus: a possible pain center. J Neurophysiol 45:443-465.

Dado RJ, Giesler GJ Jr (1990) Afferent input to nucleus submedius in rats: retrograde labeling of neurons in the spinal cord and caudal medulla. J Neurosci 10:2672-2686.

Driessen AK, Farrell MJ, Mazzone SB, McGovern AE (2015) The role of the paratrigeminal nucleus in vagal afferent evoked respiratory reflexes: a neuroanatomical and functional study in guinea pigs. Front Physiol 6:378.

Driessen AK, Farrell MJ, Mazzone SB, McGovern AE (2016) Multiple neural circuits mediating airway sensations: recent advances in the neurobiology of the urge-to-cough. Respir Physiol Neurobiol 226:115-120.

Driessen AK, McGovern AE, Behrens R, Moe AAK, Farrell MJ, Mazzone SB (2020) A role for neurokinin 1 receptor expressing neurons in the paratrigeminal nucleus in bradykinin-evoked cough in guinea-pigs. J Physiol 598:2257-2275.

Dubner R, Ren K (2013) Descending modulation of nociceptive processing. In: Encyclopedia of pain (Gebhart GF, Schmidt RF, eds), pp 59-268. Berlin, Heidelberg: Springer.

Farrell MJ, Cole LJ, Chiapoco D, Egan GF, Mazzone SB (2012) Neural correlates coding stimulus level and perception of capsaicin-evoked urge-tocough in humans. Neuroimage 61:1324-1335.

Farrell MJ, Bautista TG, Liang E, Azzollini D, Egan GF, Mazzone SB (2020) Evidence for multiple bulbar and higher brain circuits processing sensory inputs from the respiratory system in humans. J Physiol. Advance online publication. Retrieved October 7, 2020. doi: 10.1113/JP280220.

Fu JJ, Tang JS, Yuan B, Jia H (2002) Response of neurons in the thalamic nucleus submedius $(\mathrm{Sm})$ to noxious stimulation and electrophysiological identification of on- and off-cells in rats. Pain 99:243-251.

Hardy SG (1975) Analgesia elicited by prefrontal stimulation. Brain Res 339:281-284.

Hewitt MM, Adams G Jr, Mazzone SB, Mori N, Yu L, Canning BJ (2016) Pharmacology of bradykinin-evoked coughing in guinea pigs. J Pharmacol Exp Ther 357:620-628.

Huo FQ, Chen T, Lv BC, Wang J, Zhang T, Qu CL, Li YQ, Tang JS (2009) Synaptic connections between GABAergic elements and serotonergic terminals or projecting neurons in the ventrolateral orbital cortex. Cereb Cortex 19:1263-1272.

Jia H, Xie YF, Xiao DQ, Tang JS (2004) Involvement of GABAergic modulation of the nucleus submedius $(\mathrm{Sm})$ morphine-induced antinociception. Pain 108:28-35.

Kalia M, Richter D (1988) Rapidly adapting pulmonary receptor afferents: II. Fine structure and synaptic organization of central terminal processes in the nucleus of the tractus solitarius. J Comp Neurol 274:574-594.

Kawakita K, Dostrovsky JO, Tang JS, Chiang CY (1993) Responses of neurons in the rat thalamic nucleus submedius to cutaneous, muscle and visceral nociceptive stimuli. Pain 55:327-338.

Kawakita K, Sumiya E, Murase K, Okada K (1997) Response characteristics of nucleus submedius neurons to colo-rectal distension in the rat. Neurosci Res 28:59-66.

Kim J-H, Gangadharan G, Byun J, Choi E-J, Lee CJ, Shin H-S (2018) Yinand-yang bifurcation of opioidergic circuits for descending analgesia at the midbrain of the mouse. Proc Natl Acad Sci U S A 115:11078-11083. 
Kim SH, Hadley SH, Maddison M, Patil M, Cha B, Kollarik M, Taylor-Clark TE (2020) Mapping of sensory nerve subsets within the vagal ganglia and the brainstem using reporter mice for Pirt, TRPV1, 5-HT3, and Tac1 expression. eNeuro 7:ENEURO.0494-19.2020.

Kubin L, Alheid GF, Zuperku EJ, McCrimmon DR (2006) Central pathways of pulmonary and lower airway vagal afferents. J Appl Physiol (1985) 101:618-627.

Kupari J, Häring M, Agirre E, Castelo-Branco G, Ernfors P (2019) An atlas of vagal sensory neurons and their molecular specialization. Cell Rep 27:2508-2523.e4.

Kuramoto E, Iwai H, Yamanaka A, Ohno S, Seki H, Tanaka YR, Furuta T, Hioki H, Goto T (2017) Dorsal and ventral parts of thalamic nucleus submedius project to different areas of rat orbitofrontal cortex: a single neuron-tracing study using virus vectors. J Comp Neurol 525:3821-3839.

Lee M, Manders TR, Eberle SE, Su C, D'amour J, Yang R, Lin HY, Deisseroth K, Froemke RC, Wang J (2015) Activation of corticostriatal circuitry relieves chronic neuropathic pain. J Neurosci 35:5247-5259.

Leech J, Mazzone SB, Farrell MJ (2012) The effect of placebo conditioning on capsaicin-evoked urge to cough. Chest 142:951-957.

Leech J, Mazzone SB, Farrell MJ (2013) Brain activity associated with placebo suppression of the urge-to-cough in humans. Am J Respir Crit Care Med 188:1069-1075.

Ma W, Peschanski M, Ohara LPT (1988) Fine structure of the dorsal part of the nucleus submedius of the rat thalamus: an anatomical study with reference to possible pain pathways. Neuroscience 26:147-159.

Mahadi KM, Lall VK, Deuchars SA, Deuchars J (2019) Cardiovascular autonomic effects of transcutaneous auricular nerve stimulation via the tragus in the rat involve spinal cervical sensory afferent pathways. Brain Stimul 12:1151-1158.

Mazzone SB, Canning BJ (2002) Synergistic interactions between airway afferent nerve subtypes mediating reflex bronchospasm in guinea pigs. Am J Physiol Regul Integr Comp Physiol 283:R86-R98.

Mazzone SB, Undem BJ (2016) Vagal afferent innervation of the airways in health and disease. Physiol Rev 96:975-1024.

Mazzone SB, McGovern AE, Koo K, Farrell MJ (2009) Mapping supramedullary pathways involved in cough using functional brain imaging: comparison with pain. Pulm Pharmacol Ther 22:90-96.

Mazzone SB, Tian L, Moe AAK, Trewella MW, Ritchie ME, McGovern AE (2020) Transcriptional profiling of individual airway projecting vagal sensory neurons. Mol Neurobiol 57:949-963.

McAllen RM, Shafton AD, Bratton BO, Trevaks D, Furness JB (2018) Calibration of thresholds for functional engagement of vagal A, B and C fiber groups in vivo. Bioelectron Med (Lond) 1:21-27.

McGovern AE, Davis-Poynter N, Farrell MJ, Mazzone SB (2012) Transneuronal tracing of airways-related sensory circuitry using herpes simplex virus 1, strain H129. Neuroscience 207:148-166.

McGovern AE, Driessen AK, Simmons DG, Powell J, Davis-Poynter N, Farrell MJ, Mazzone SB (2015a) Distinct brainstem and forebrain circuits receiving tracheal sensory neuron inputs revealed using a novel conditional anterograde transsynaptic viral tracing system. J Neurosci 35:7041-7055

McGovern AE, Davis-Poynter N, Yang S-K, Simmons DG, Farrell MJ, Mazzone SB (2015b) Evidence for multiple sensory circuits in the brain arising from the respiratory system: an anterograde viral tract tracing study in rodents. Brain Struct Funct 220:3683-3699.

Millan M (2002) Descending control of pain. Prog Neurobiol 66:355-474.

Morice AH, Kitt MM, Ford AP, Tershakovec AM, Wu WC, Brindle K, Thompson R, Thackray-Nocera S, Wright C (2019) The effect of gefapixant, a P2X3 antagonist, on cough reflex sensitivity: a randomised placebo-controlled study. Eur Respir J 54:1900439.

Multon S, Schoenen J (2005) Pain control by vagus nerve stimulation: from animal to man... and back. Acta Neurol Belg 105:62-67.

Nassenstein C, Taylor-Clark TE, Myers AC, Ru F, Nandigama R, Bettner W, Undem BJ (2010) Phenotypic distinctions between neural crest and placodal derived vagal C-fibres in mouse lungs. J Physiol 588:4769-4783.

Nonomura K, Woo S-H, Chang RB, Gillich A, Qiu Z, Francisco AG, Ranade SS, Liberles SD, Patapoutian A (2017) Piezo2 senses airway stretch and mediates lung inflation-induced apnoea. Nature 541:176-181.

Ong W-Y, Christian S, Stohler CS, Deron R, Herr DR (2019) Role of the prefrontal cortex in pain processing. Mol Neurobiol 56:1137-1166.

Ossipov MH, Morimura K, Porreca F (2014) Descending pain modulation and chronification of pain. Curr Opin Support Palliat Care 8:143-151.

Randich A, Gebhart GF (1992) Vagal afferent modulation of nociception. Brain Res Brain Res Rev 17:77-99.

Ren K, Zhuo M, Randich A, Gebhart GF (1993) Vagal afferent stimulationproduced effects on nociception in capsaicin-treated rats. J Neurophysiol 69:1530-1540.

Schweinhardt P, Bushnell MC (2010) Pain imaging in health and diseasehow far have we come? J Clin Invest 120:3788-3797.

Straube A, Ellrich J, Eren O, Blum B, Ruscheweyh R (2015) Treatment of chronic migraine with transcutaneous stimulation of the auricular branch of the vagal nerve (auricular t-VNS): a randomized, monocentric clinical trial. J Headache Pain 16:543.

Tang JS, Qu CL, Huo FQ (2009) The thalamic nucleus submedius and ventrolateral orbital cortex are involved in nociceptive modulation: a novel pain modulation pathway. Prog Neurobiol 89:383-389.

Wu Y, Fu D, Gu Q, Li Y, Qian Z, Han J, Liu Z, Ren W, Liu Y (2020) Activation of $\mathrm{CB} 1$ receptors on GABAergic interneurons in the ventrolateral orbital cortex induces analgesia. Neurosci Lett 736:135286.

Xia L, Leiter JC, Bartlett D Jr (2008) Laryngeal apnea in rat pups: effects of age and body temperature. J Appl Physiol (1985) 104:269-274.

Xiao DQ, Tang JS, Yuan B, Jia H (1999) Inhibitory effects of 5-hydroxytryptamine microinjection into thalamic nucleus submedius on rat tail flick reflex are mediated by 5-HT2 receptors. Neurosci Lett 260:85-88.

Xiao DQ, Zhu JX, Tang JS, Jia H (2005a) 5-hydroxytryptamine 1A (5-HT1A) but not 5-HT3 receptor is involved in mediating the nucleus submedius 5-HT-evoked antinociception in the rat. Brain Res 1046:38-44.

Xiao DQ, Zhu JX, Tang JS, Jia H (2005b) GABAergic modulation mediates antinociception produced by serotonin applied into thalamic nucleus submedius of the rat. Brain Res 1057:161-167.

Yoshida A, Dostrovsky JO, Sessle BJ, Chiang CY (1991) Trigeminal projections to the nucleus submedius of the thalamus in the rat. J Comp Neurol 307:609-625.

Yoshida A, Dostrovsky JO, Chiang CY (1992) The afferent and efferent connections of the nucleus submedius in the rat. J Comp Neurol 324:115133.

Zhang S, Tang JS, Yuan B, Jia H (1998) Inhibitory effects of glutamateinduced activation of thalamic nucleus submedius are mediated by ventrolateral orbital cortex and periaqueductal gray in rats. Eur J Pain 2:153163.

Zhang S, Tang JS, Yuan B, Jia H (1999) Electrically-evoked inhibitory effects of the nucleus submedius on the jaw-opening reflex are mediated by ventrolateral orbital cortex and periaqueductal gray matter in the rat. Neuroscience 92:867-875 